The AstrophysiCAL JournaL, 555:301-321, 2001 July 1

(C) 2001. The American Astronomical Society. All rights reserved. Printed in U.S.A.

\title{
STAR FORMATION THRESHOLDS IN GALACTIC DISKS ${ }^{1}$
}

\author{
Crystal L. Martin \\ Astronomy Department, California Institute of Technology, MC 105-24, Pasadena, CA 91125 \\ AND \\ ROBERT C. KENNICUTT, JR. \\ Steward Observatory, University of Arizona, Tucson, AZ 85721 \\ Received 2000 December 21; accepted 2001 March 5
}

\begin{abstract}
We report the first results of a detailed study of the star formation law in a sample of 32 nearby spiral galaxies with well-measured rotation curves, $\mathrm{H}$ I and $\mathrm{H}_{2}$ (as traced by $\mathrm{CO}$ ) surface density profiles, and new $\mathrm{H} \alpha \mathrm{CCD}$ photometry. In this paper we present an atlas of $\mathrm{H} \alpha$ images and radial surface brightness profiles and describe a surface density threshold in the star formation law. Prominent breaks in the $\mathrm{H} \alpha$ surface brightness profiles are identified in nearly all of the actively star-forming disks, confirming previous claims of star formation thresholds based on lower quality data. We measure the ratio of the gas density to the critical density for local gravitational stability at the threshold radii. The outer threshold radii observed in Sab-Sdm galaxies are in general agreement with those expected from the Toomre $Q$ stability criterion, confirming earlier work, but with a significant variation that appears to be weakly correlated with galaxy type. Such a trend could plausibly reflect variations in the relative contribution of the stellar disk to the instability of the gas disk across this range of galaxy types. Among disks with subcritical gas surface densities, and outside the threshold radius in star-forming disks, the number of isolated $\mathrm{H}$ II regions increases as the gas surface density approaches the critical density. At the thresholds, the gas surface densities span a wide range, and the atomic/molecular gas fraction is highest in the disks having the lowest total gas surface density. The simple Toomre condition fails to account for the active star formation in the inner disks of low-mass spirals such as NGC 2403 and M33. An alternative stability criterion based on the shear in the disk provides a better description of these disks but is a less accurate indicator of the outer edges of star-forming disks than the Toomre criterion. These results strongly support the view that the formation of gravitationally bound interstellar clouds regulates the onset of widespread star formation, at least in the outer regions of galactic disks.
\end{abstract}

Subject headings: galaxies: evolution — galaxies: ISM — galaxies: stellar content - stars: formation

\section{INTRODUCTION}

The evolution of galaxies is strongly influenced by how quickly gas is consumed by stars. Most simulations assume that the star formation rate (SFR) scales as a power law of the gas density, as originally proposed by Schmidt (1959), or adopt the surface density parameterization introduced by Kennicutt (1989, hereafter K89). A global correlation between SFR and gas surface density $\left(\Sigma_{\mathrm{SFR}} \propto \mu_{\mathrm{gas}}^{1.4}\right)$ is obtained empirically when both quantities are normalized by the area of the stellar disk. Since galaxies typically have gaseous disks that are significantly larger than their stellar disks (e.g., Warmels 1988a, 1988b; Gallagher \& Hunter 1984), the gas in the outer disk is not included in the surface density measurement (Kennicutt 1998). The empirical star formation recipe implicitly includes a surface density threshold for star formation, and this should be incorporated in the prescriptions used in numerical simulations.

The idea that gravitational instability might determine the critical gas density for star formation was introduced by Spitzer (1968) and Quirk (1972) soon after local stability criteria for differentially rotating disks were developed (Toomre 1964; Goldreich \& Lynden-Bell 1965). A thin, gas disk is unstable to axisymmetric disturbances where the

\footnotetext{
${ }^{1}$ Data obtained in part at Kitt Peak National Observatory, operated by AURA under contract to the National Science Foundation.
}

Toomre $Q$ parameter,

$$
Q(R) \equiv \frac{\sigma \kappa}{\pi G \mu},
$$

is less than unity. The epicyclic frequency, $\kappa$, velocity dispersion, $\sigma$, and surface density, $\mu$, refer to the gas disk at galactocentric radius $R$. Widespread star formation is expected where the gas surface density exceeds the critical surface density defined as

$$
\mu_{\text {crit }}=\alpha_{Q} \frac{\sigma \kappa}{\pi G} .
$$

The parameter $\alpha_{Q}$ is fitted to the threshold values of the radially varying quantity

$$
\alpha(R)=\frac{\mu_{\mathrm{gas}}(R)}{\mu_{\mathrm{crit}}(R)}
$$

and makes allowances for deviations from the idealized thin-disk model such as finite scale height or the presence of a stellar disk. The velocity dispersion is predicted to remain roughly constant in self-regulated regions of disks (Silk 1997) and may have a lower bound set by the dissipation of MHD-driven turbulence in the outer disk (Sellwood \& Balbus 1999). If the gas velocity dispersion does not vary much with radius across a spiral galaxy, then the critical surface density falls as roughly $R^{-1}$. This gradient is 
shallower than the decline in the total gas surface density with radius, so the critical density will exceed the gas surface density at some threshold radius. Gravitational stability therefore provides an appealing explanation for the rather sharp edges of stellar disks (van der Kruit \& Searle 1981a, 1981b, 1982a, 1982b).

K89 directly tested this hypothesis using the radial distribution of $\mathrm{H}$ II regions to trace the SFR. He adopted a constant gas velocity dispersion of $6 \mathrm{~km} \mathrm{~s}^{-1}$ and found that the Schmidt law broke down at radii where the gas surface density was less than $0.63 \mu_{\text {crit }}\left(\mathrm{K} 89^{2}\right)$. The gas surface density in low surface brightness galaxies was subsequently shown to be below the critical density for gravitational instability, and gravitational thresholds were used to explain the low level of star formation activity in these gasrich galaxies (van der Hulst et al. 1993; van Zee et al. 1996, 1997). More recently, Ferguson et al. (1998) have questioned the validity of a constant gas velocity dispersion. Since measurements of the $\mathrm{H}$ I velocity dispersion demand both high angular resolution and high brightness sensitivity, requirements not met by most observations, the most feasible way forward currently is a comparison of measured threshold radii to the thresholds predicted using constant velocity dispersion in a large sample of galaxies.

Hunter, Elmegreen, \& Baker (1998, hereafter HEB98) have recently questioned the utility of the Toomre criterion for describing star formation thresholds. They measure a mean value of $\alpha_{Q}$ in irregular galaxies that is a factor of 2 lower than that found by K89 for spiral galaxies. This result implies that the gas in the irregular galaxies is less stable than the gas in spiral galaxies. In addition to the absence of spiral density waves in irregular galaxies, these disks have less rotational shear on average (i.e., their rotation curves are closer to solid body). If the local shear rate, rather than the Coriolis force (essentially $\kappa$ ), best describes the destruction rate of giant clouds, then the stability criterion would need to be modified (Elmegreen 1987, 1991, 1993). For example, the local shear rate is described by the Oort $A$ constant,

$$
A=-0.5 R \frac{d \Omega}{d R} .
$$

Following Elmegreen (1993) and HEB98, shearing perturbations grow at the rate $\pi G \mu / \sigma$, and the critical column density for significant growth in the presence of shear becomes

$$
\mu_{\text {crit }}^{A} \approx \frac{\alpha_{A} A \sigma}{\pi G},
$$

and $\alpha_{A} \approx 2.5$. This surface density threshold is extremely low in the inner regions of galaxies with slowly rising rotation curves but approaches the Toomre stability condition where the rotation speed is constant.

In addition to this degeneracy, many other descriptions of star formation thresholds, including gas deficiencies in the outer disk (Warmels 1988a; Cayatte et al. 1990), the phase structure of the interstellar gas (Elmegreen \& Parravao 1994), or the radiative feedback from young stars, have been advanced (Federman, Glassgold, \& Kwan 1979; Skillman 1987). With such a broad array of physical processes at work, the K89 analysis should be repeated on a larger, more

\footnotetext{
${ }^{2} \mathrm{~K} 89$ reported a value of $\alpha_{Q}=0.67$ but used a constant of 3.36 rather than $\pi$ in eq. (1).
}

diverse sample of galaxies. The $\mathrm{H} \alpha$ surface brightness profiles presented in this paper supersede those in the K89 paper, which were based largely on $\mathrm{H}$ II region counts. The $\mathrm{H} \alpha$ images presented alongside the azimuthally averaged profiles illustrate the sharp decline in the covering fraction of $\mathrm{H}$ II regions that defines the threshold radius. Finally, the number of galaxies with published $\mathrm{CO}$ observations has greatly increased in the last decade, in large part because of the Five College Radio Astronomy Observatory (FCRAO) CO survey (Young et al. 1995). Hence, it is now possible to examine the full range of spiral galaxy types using atomic and molecular gas data compiled from the published literature.

The paper is organized as follows. In $\S 2$ we describe our $\mathrm{H} \alpha$ observations and the atomic and molecular gas data used in our new analysis. In $\S 3$ we identify the star formation thresholds, measure gas densities at the thresholds, and evaluate the Toomre stability criterion. In $\S 4$ we discuss the failures of the model, the shear criterion for stability, the influence of the stellar disk on the stability criterion, and the implications for the global SFR. The data presented here will be used to discuss the processes regulating the SFR in the high-density regime (i.e., where gas surface density exceeds the threshold density) in a subsequent paper.

\section{DATA}

We selected 32 spiral galaxies divided roughly evenly between types $\mathrm{Sab}$ and $\mathrm{Sdm}$ that had published ${ }^{12} \mathrm{CO}$ $(J=1-0)$ and $21 \mathrm{~cm}$ radial intensity profiles. Nearly half the galaxies are Virgo Cluster members, and 15 of the galaxies are classified as barred galaxies in the RC1 (de Vaucoulers system). In the K89 paper, radial profiles were estimated for 16 galaxies from $\mathrm{H}$ iI region counts. That subsample was dominated by type $\mathrm{Sc}$ galaxies.

Table 1 lists the type, distance, and disk inclination for each galaxy in our sample. The critical density of a disk scales inversely with the assumed distance and is the only distance-dependent quantity that enters our analysis. Most of the distances come from measurements of Cepheid variables. We correct surface brightness, surface density, and rotation speed measurements for disk inclination. Disk inclinations and position angle were generally adopted from $21 \mathrm{~cm}$ observations. If these values were heavily weighted by $\mathrm{H}$ I beyond the optical disk, we used the shape of the optical continuum isophotes to derive a disk inclination and position angle. The position angles are given in Table 1 and describe the major axis of elliptical apertures used in our $\mathrm{H} \alpha$ photometry.

\section{1. $\mathrm{H} \alpha$ Imaging}

We use recombination line radiation from hydrogen to trace the location of young, massive stars in the disks. The $\mathrm{H} \alpha$ line was chosen for the imaging because only optical CCD cameras offered a wide enough field of view to cover the entire disk in one or two pointings per galaxy. The measured $\mathrm{H} \alpha$ luminosities can be directly converted to SFRs using the calibration of Kennicutt, Tamblyn, \& Congdon (1994):

$$
\operatorname{SFR}\left(M_{\odot} \mathrm{yr}^{-1}\right)=\frac{L_{\mathrm{H} \alpha}}{1.25 \times 10^{41} \mathrm{ergs} \mathrm{s}^{-1}} 10^{0.4 A(\mathrm{H} \alpha)} .
$$

This relation was derived using a Salpeter initial mass function, $d N(m) / d m=m^{-2.35}$, for stellar masses between $m=0.1$ and $100 M_{\odot}$. The stars were evolved along the 
TABLE 1

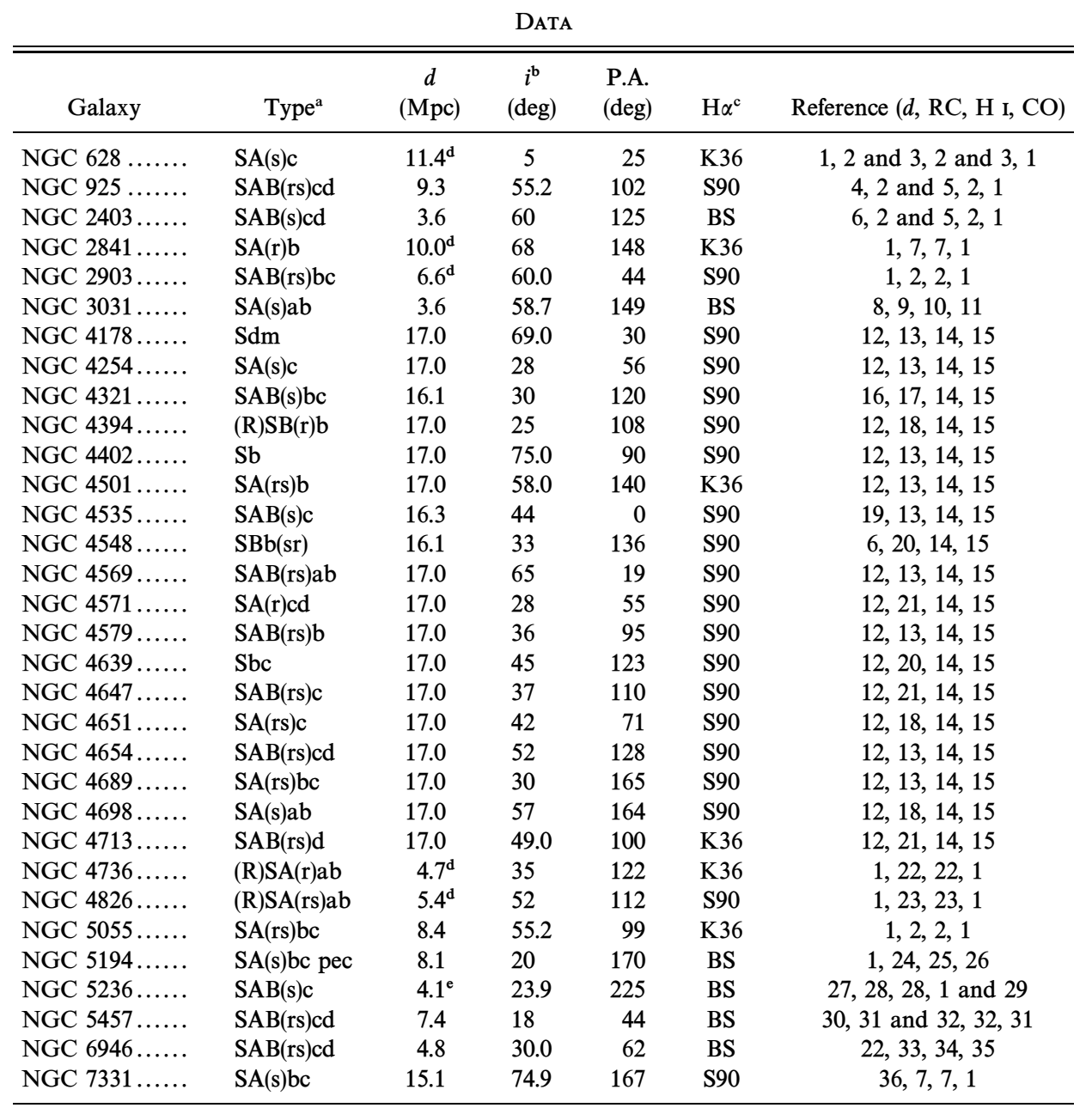

\footnotetext{
a de Vaucouleurs type from RC1.

${ }^{\mathrm{b}}$ Inclination of the disk such that a face-on orientation is $0^{\circ}$. (S90)

' Telescope used for H $\alpha$ observation: Burrell-Schmidt (BS); KPNO 36 inch (K36); Steward 90 inch

d Published distance adjusted to $H_{0}=70 \mathrm{~km} \mathrm{~s}^{-1} \mathrm{Mpc}^{-1}$.

${ }^{\mathrm{e}}$ Distance to M83 group.

REFERENCES - (1) Young et al. 1995. (2) Wevers et al. 1986. (3) Kamphuis \& Briggs 1992. (4) Silbermann et al. 1996. (5) Pisano, Wilcots, \& Elmegreen 1998. (6) Graham et al. 1999. (7) Bosma 1981. (8) Freedman et al. 1994a. (9) Adler \& Westpfahl 1996. (10) Rots 1975. (11) Sage \& Westpfahl 1991. (12) Freedman et al. 1994b. (13) Guhathakurta et al. 1988. (14) Warmels 1988c. (15) Kenney \& Young 1988. (16) Ferrarese et al. 1996. (17) Sofue 1997. (18) Warmels 1988a. (19) Macri et al. 1999. (20) Rubin et al. 1999. (21) Warmels 1988b. (22) Bosma 1977. (23) Braun et al. 1994. (24) Sofue 1996. (25) Tilanus \& Allen 1991. (26) Lord \& Young 1990. (27) Saha et al. 1995. (28) Tilanus \& Allen 1993. (29) P. Crosthwaite et al. 2001, in preparation. (30) Kelson et al. 1996. (31) Kenney et al. 1991. (32) Bosma et al. 1981. (33) Carignan et al. 1990. (34) Tacconi \& Young 1986. (35) Tacconi \& Young 1989. (36) Hughes et al. 1998.
}

evolutionary tracks of Schaller et al. (1992), and the ionizing flux was calculated from R. L. Kurucz (1992, private communication) model atmospheres. The amount of extinction at $\mathrm{H} \alpha, A_{\mathrm{H} \alpha}=0.78 A_{V}$ (Miller \& Mathews 1972), is the largest source of uncertainty in the measured SFR.

Narrowband continuum and $\mathrm{H} \alpha$ images were obtained at the Steward Observatory Bok telescope, KPNO BurrellSchmidt telescope, and KPNO 36 inch telescope. These CCD frames were corrected for fixed pattern noise in the standard way using a bias frame, dome flat, and sky flat. The 90 inch data were obtained with a focal reducer, and scattered light produced ghost images that had to be removed in the background subtraction. Foreground stars were used to register the images and subtract the continuum emission from each narrowband $\mathrm{H} \alpha$ image. The line flux was then corrected for atmospheric extinction and the transmission of the filter at $\mathrm{H} \alpha$ relative to the average transmission over the bandpass. Data obtained at the Bok telescope and Burrell-Schmidt telescope were flux calibrated using observations of standard stars. The five galaxies observed at the Kitt Peak 36 inch and NGC 7331, NGC 925, NGC 628, and NGC 4402 were calibrated from the integrated fluxes of Kennicutt \& Kent (1983) or Kennicutt (1998). The Burrell-Schmidt images do not include [N II] emission, so we increased their flux by a spectroscopically determined factor ranging from 1.2 to 1.4 to include [N II] emission consistently. Figure 1 compares our $\mathrm{H} \alpha+[\mathrm{N} \mathrm{II}]$ $\lambda \lambda 6548,83$ fluxes to previously published work. The independent measurements are typically consistent to within $15 \%$.

The radial distribution of $\mathrm{H} \alpha$ emission was measured from surface photometry. The optical nucleus of each 


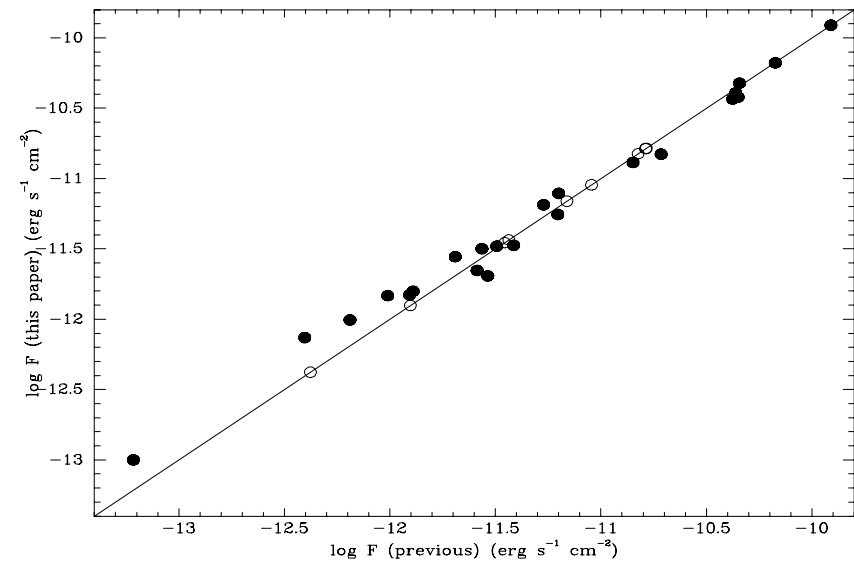

FIG. 1.-Integrated $\mathrm{H} \alpha+[\mathrm{N}$ II] fluxes vs. published values of Kennicutt \& Kent (1983) and Kennicutt (1998). Open symbols denote galaxies with dependent calibrations, i.e., the data presented here were calibrated using the data plotted along the abscissa.

galaxy defines the center of a set of concentric, elliptical apertures. The width of the annuli is a trade-off between the desire to average over individual $\mathrm{H}$ iI regions and the need to measure breaks in the profile accurately. A width of about $10^{\prime \prime}$ proved to be a good compromise for most of the galaxies. The profiles changed very little if this width was changed by a factor of 2 . The profiles were corrected to face-on orientation.

To convert the surface brightness profiles in Figure 3 to SFR per unit area, we recommend applying an internal extinction correction, $A_{\mathrm{H} \alpha}=1.1 \mathrm{mag}$, derived from comparison of the free-free radio emission and the $\mathrm{H} \alpha$ emission from spiral galaxies (e.g., van der Hulst et al. 1988). Correction for $[\mathrm{N}$ II] emission in the bandpass reduces the SFR by $15 \%-30 \%$. Radial gradients in internal extinction are typically not strong compared to the scatter in extinction at a given radius (e.g., Zaritsky, Kennicutt, \& Huchra 1994; Webster \& Smith 1983; McCall, Rybski, \& Shields 1985). An exception is the nuclear region. The central SFRs implied by these profiles are highly uncertain as a result of the unknown amount of dust obscuration, photoionization by active nuclei, and continuum subtraction errors.

The limiting surface brightness was estimated from the uncertainty in the background and the area of the aperture and then verified by adding fake sources to the images. Where the covering factor of $\mathrm{H}$ II regions was low, photometry could be obtained for individual $\mathrm{H}$ II complexes using local background measurements. In other regions, variations in the mean background level dominate the uncertainty in the photometry. In particular, scattered light in the focal reducer camera at the Bok telescope produced ghost images that were easily identified by eye but difficult to model and subtract. We used a mean background measurement and then estimated the maximum error using the full range of mean background levels on the frame. The error bars shown on the radial profiles are therefore maxima and minima in these regions.

\subsection{Molecular Gas Surface Densities}

Only a few galaxies in the sample have been completely mapped in the ${ }^{12} \mathrm{CO}(J=1-0)$ line. FCRAO maps have been published for NGC 5194 (Lord \& Young 1990) and NGC 6946 (Tacconi \& Young 1989). The galaxies NGC 5457 (Kenney, Scoville, \& Wilson 1991), NGC 3031 (Sage \&
Westphal 1991), NGC 5236 (P. Crosthwaite \& J. Turner 2001, in preparation), and the inner $3.2 \mathrm{kpc}$ of NGC 2403 (Thornley \& Wilson 1995) have been mapped with the NRAO $12 \mathrm{~m}$ telescope. For the rest of our sample, the CO radial profiles represent measurements made along the major axis of each galaxy. These profiles have a resolution of $\sim 45^{\prime \prime}$. The data are described in detail in survey papers by Kenney \& Young (1988) and Young et al. (1995).

These source papers adopt CO-to- $\mathrm{H}_{2}$ conversion factors based on Galactic calibrations. We follow this convention but adopt a common scale of

$$
N\left(\mathrm{H}_{2}\right)=\left(2.8 \times 10^{20} \mathrm{~cm}^{-2}\right) I_{\mathrm{CO}}\left(K\left(T_{R}\right) \mathrm{km} \mathrm{s}^{-1}\right)
$$

(Bloemen et al. 1986) for all the galaxies. The beamaveraged brightness temperature, $T_{R}$, inferred from the measured antenna temperature depends on the source beam coupling efficiency and the forward scattering and spillover efficiency as described, for example, by Kenney \& Young (1988) and Young et al. (1995). We adopted their calibrations, $\eta_{\mathrm{fss}}=0.75$ and $\eta_{c}=0.73$, for the FCRAO survey data. The NGC 5194 profile uses $\eta_{\mathrm{fss}}=0.70$ and $\eta_{c}=$ $0.69-0.81$ following the model of Lord \& Young (1990). The NRAO $12 \mathrm{~m}$ source beam coupling efficiency derived for M101 (Kenney et al. 1991) was applied to the M81 data. The $\mathrm{H}_{2}$ gas surface density was multiplied by a factor of $1.4 \cos (i)$ to correct for inclination and to include the $\mathrm{He}$ mass.

\subsection{Atomic Gas Surface Densities}

The $\mathrm{H}$ I radial profiles were taken from $21 \mathrm{~cm}$ observations listed in Table 1. The profiles from Warmels (1988c) were derived from strip scans across each galaxy with the Westerbork Synthesis Radio Telescope (WRST). The halfpower beamwidth was about $12^{\prime \prime}$ in right ascension by $12^{\prime \prime}(\sin \delta)^{-1}$ in declination. These scans are essentially major axis profiles for many of the galaxies, but the scans are aligned closer to the minor axis of NGC 4178, NGC 4321, NGC 4501, NGC 4535, NGC 4548, and NGC 4689.

The other profiles were obtained by integrating over ellipses and correcting to face-on orientation. The typical half-power beamwidth of the other WRST maps is $30^{\prime \prime}-60^{\prime \prime}$ (Wevers, van der Kruit, \& Allen 1986; Bosma 1981; Bosma, van der Hulst, \& Sullivan 1977; Bosma, Goss, \& Allen 1981; Rots 1975). The WSRT observations of NGC 5194 (Tilanus \& Allen 1991) and NGC 5236 (Tilanus \& Allen 1993) have higher resolution. The surface density profile for NGC 6946 comes from a Very Large Array (VLA) map (Tacconi \& Young 1986) with 40" resolution. Braun et al. (1994) describe the compilation of WRST and VLA observations used to construct the high-resolution $\mathrm{H}$ I map of NGC 4826.

\subsection{Gas Kinematics}

Rotation curves are needed to calculate radial stability profiles for each galaxy. Our analysis uses the H I $21 \mathrm{~cm}$ rotation curves derived by Wevers et al. (1986) and Guhathakurta (1988) and position-velocity diagrams published by Warmels (1988a, 1988b). Velocities measured from CO observations were included in the rotation curves for NGC 5194, NGC 5457, and NGC 4321 (Kenney \& Young 1988; Young et al. 1995; Sofue 1996). These CO and H I $21 \mathrm{~cm}$ observations lack sufficient spatial resolution to determine the kinematics in the inner $\sim 45^{\prime \prime}$ of the disks. Optical emission-line velocities were used to improve inner rotation curves of NGC 4548 and NGC 4639 (Rubin, Waterman, \& 
Kenney 1999). In general, the dynamics of the inner disk is not well constrained by the observations compiled here, and our stability analysis will focus on the outer regions of disks.

Measurements of the $\mathrm{H}$ I velocity dispersion are difficult to make in galaxies that are inclined enough (relative to our sight line) to model their rotation curves accurately. Studies of spiral galaxies find $\sigma=3-10 \mathrm{~km} \mathrm{~s}^{-1}$ in the outer disk (van der Kruit \& Shostak 1984; Dickey, Hanson, \& Helou 1990). A similar range is estimated for dwarf irregular galaxies: 7.6-11.4 $\mathrm{km} \mathrm{s}^{-1}$ (van Zee et al. 1997). The higher velocity dispersions tend to be associated with the regions of higher star formation activity (Shostak \& van der Kruit 1984; Jogee 1999). We adopt $\sigma=6 \mathrm{~km} \mathrm{~s}^{-1}$ to facilitate comparison with K89.

\section{TESTING THE GRAVITATIONAL STABILITY MODEL AT THE STAR FORMATION THRESHOLDS}

To give the reader an appreciation of the accuracy with which the stability criterion can be evaluated, we step through our analysis of NGC 5236. Our $\mathrm{H} \alpha$ atlas is then used to illustrate further what is meant by the expression "star formation threshold." We describe the properties of the gas disks at the thresholds and evaluate the local stability of the disks using the single-fluid model.

\subsection{Case Study: NGC 5236}

Figure 2 shows that the broken spiral pattern of $\mathrm{H} \alpha$ emission from NGC 5236 creates a smooth radial surface brightness profile, which is well fitted by an exponential profile over a wide annulus. The strongest break in the radial profile defines the threshold radius, $R_{\mathrm{HI}}=305^{\prime \prime}$. The azimuthally averaged SFR there is $0.0027 M_{\odot} \mathrm{yr}^{-1} \mathrm{kpc}^{-2}$, but the best estimate for the upper limit just $500 \mathrm{pc}$ farther out is 100 times lower. The $\mathrm{H} \alpha$ surface brightness is also low in the inner disk, where the star-forming regions are concentrated along the bar, but the magnitude of the deficit relative to the fit is much smaller. In Figure 3, a few $\mathrm{H}$ II regions are seen beyond $R_{\mathrm{H} \text { I }}$, but their covering factor is much lower than in the region $R \leq R_{\mathrm{H} \text { II }}$. The threshold radius derived from the azimuthally averaged $\mathrm{H} \alpha$ profile is a good approximation to the "edge" of the star-forming disk, but it does overestimate the threshold radius at some position angles (e.g., northeast side of NGC 5236) since the shape of the $\mathrm{H} \alpha$ isophotes changes slightly with galactocentric radius.

The middle panel of Figure 2 compares the radial variation in the azimuthally averaged H I surface density (Tilanus \& Allen 1993) to the variation in molecular gas surface density along the major axis (Young et al. 1995). More recent CO mapping across NGC 5236 (P. Crosthwaite \& J. Turner 2000, private communication) shows that the molecular gas is concentrated along the bar near the major axis. We find that the spatial mismatch (one-dimensional cut vs. azimuthal averaging) exaggerates the central depletion of atomic gas relative to molecular gas. The azimuthal gas distribution is more uniform farther out in this disk. For many galaxies in our sample, complete twodimensional mapping of the gas distribution is not available. This example draws attention to the systematic errors introduced by using the one-dimensional cuts and suggests that they are significant in the inner disk when a gaseous bar is present. They do not affect the measurement of the total gas density at the threshold radius in NGC 5236. When the $\mathrm{H} \alpha$ surface brightness is plotted against the total gas density, a strong break is seen at the outer threshold.

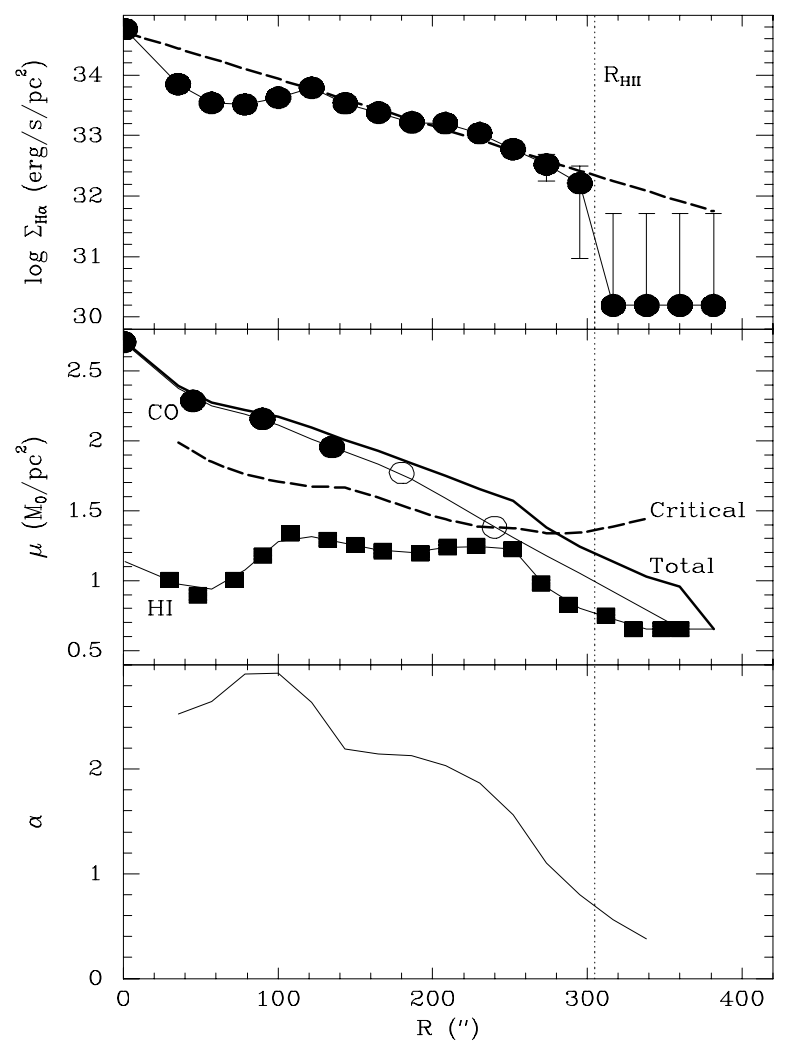

FIG. 2.-Top: $\mathrm{H} \alpha$ surface brightness profile for NGC 5236. The dashed line is the fitted exponential disk. The threshold radius is shown by the dotted line. Middle: Radial variation in gas surface density. Filled/open circles represent molecular gas density along the major axis from Young et al. (1995) and P. Crosthwaite \& J. Turner (2000, private communication). The squares show the azimuthally averaged atomic hydrogen surface density (Tilanus \& Allen 1993). (The $\mathrm{H}$ densities were multiplied by a factor of 1.4 to include the He mass.) The inner and outer spiral arms imprint the local $\mathrm{H}$ I maxima at $120^{\prime \prime}$ and $240^{\prime \prime}$. The dashed line is the critical surface density for local gravitational instability. Bottom: Ratio of total gas surface density to critical density.

This $\log \Sigma$ versus $\log \mu$ relation is well described by a power law over most of the disk, but the spatial mismatch between the atomic and molecular gas sampling in the inner disk artificially adds some structure to it.

The critical density for local gravitational instability, as defined by the Toomre $Q$ criterion, is also shown in the middle panel of Figure 2. The gas velocity dispersion is assumed to be constant, so the small-scale features reflect structure in the rotation curve of Tilanus \& Allen (1993) through the epicyclic frequency,

$$
\kappa^{2}(R)=2\left(\frac{V^{2}}{R^{2}}+\frac{V}{R} \frac{d V}{d R}\right)
$$

The rotation curves typically represent an average $V(R)$ fitted to the two-dimensional velocity field or to both sides of the major axis. The ratio of the total gas density to the critical density (Fig. 2, bottom panel) falls steadily with radius beyond the bar region. The spatial resolution of the rotation curve is insufficient to describe the critical density at radii less than $\sim 45^{\prime \prime}$. At the star formation threshold $R_{\mathrm{H} \text { II }}$, the gas density, $10 M_{\odot} \mathrm{pc}^{-2}$, is $70 \%$ of the critical density for instability $\left[\alpha\left(R_{\mathrm{H} \mathrm{II}}\right)=0.7\right]$, as given in equation (2) for $\sigma=6 \mathrm{~km} \mathrm{~s}^{-1}$. In NGC 5236, the dominant error in $\alpha\left(R_{\mathrm{H}} \mathrm{I}\right)$ is the extrapolated molecular gas density. If the 

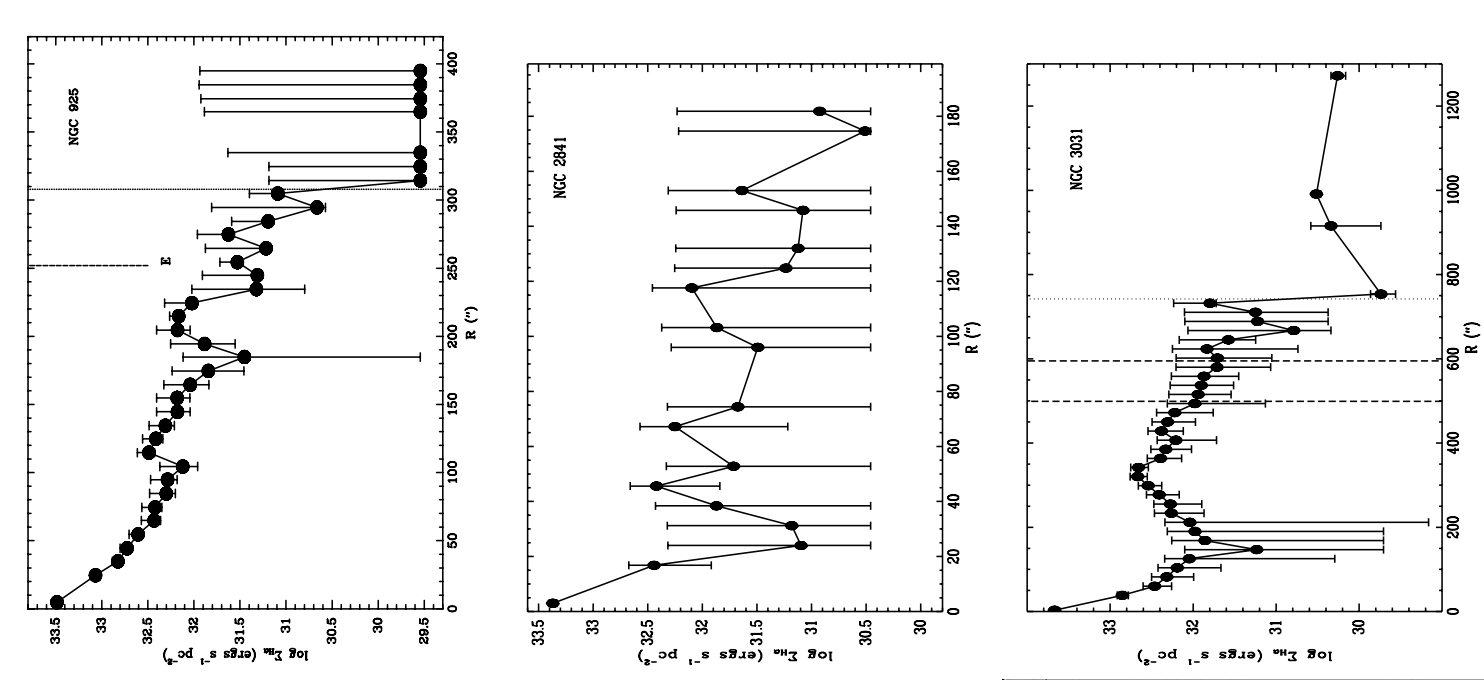

.
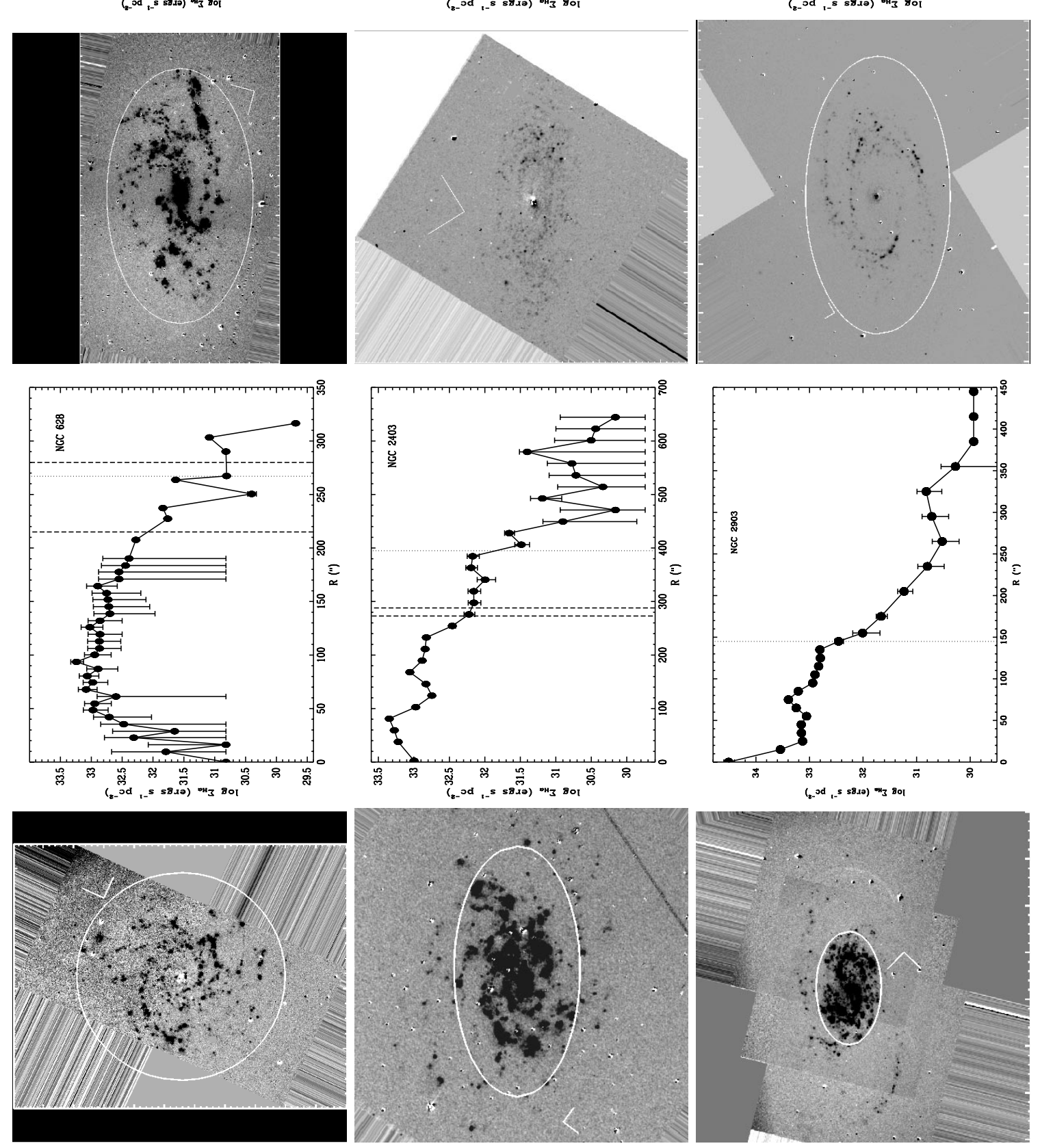

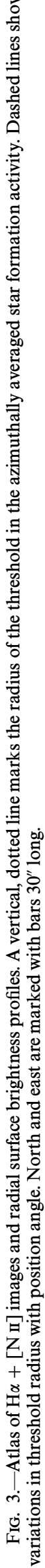



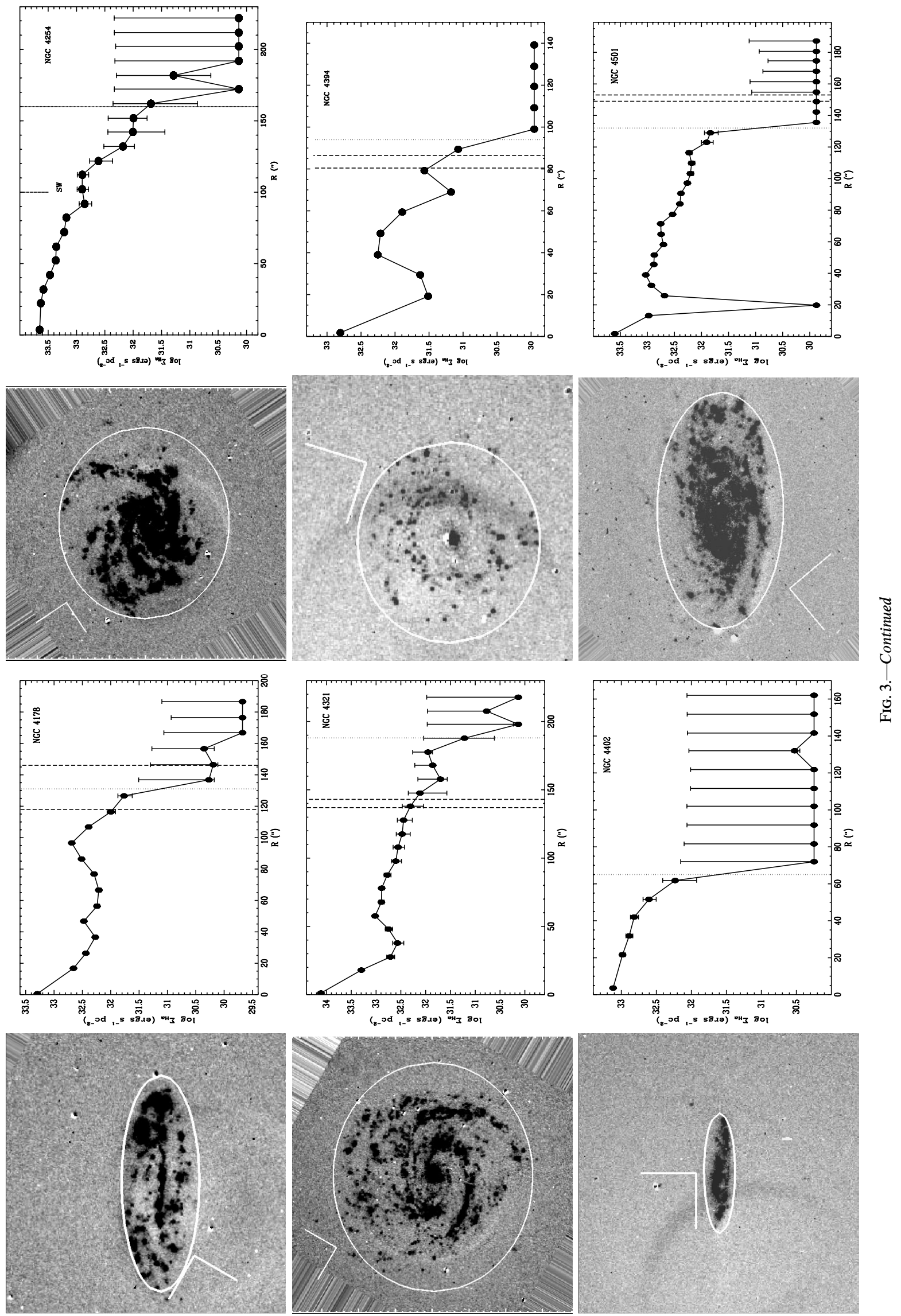

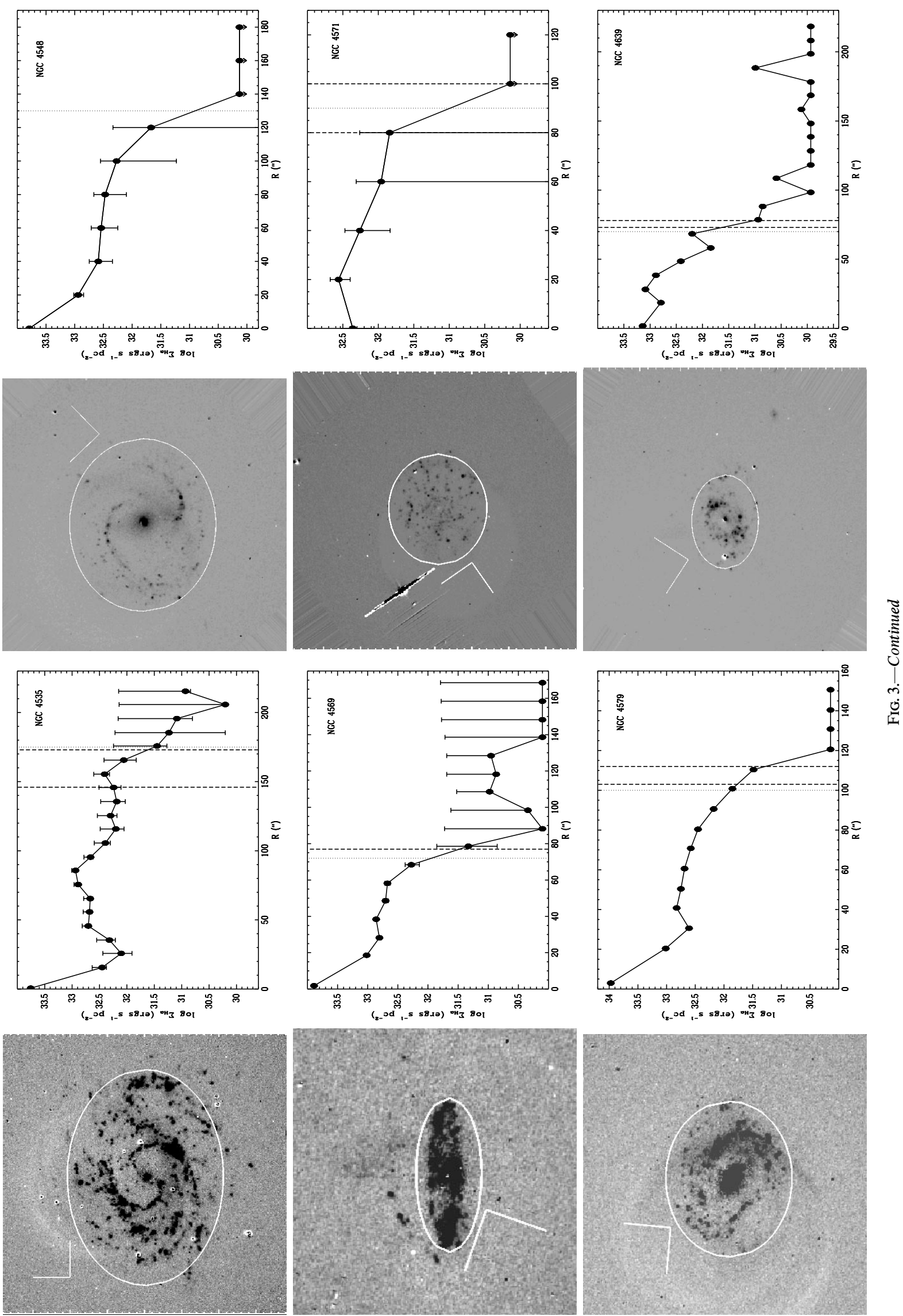

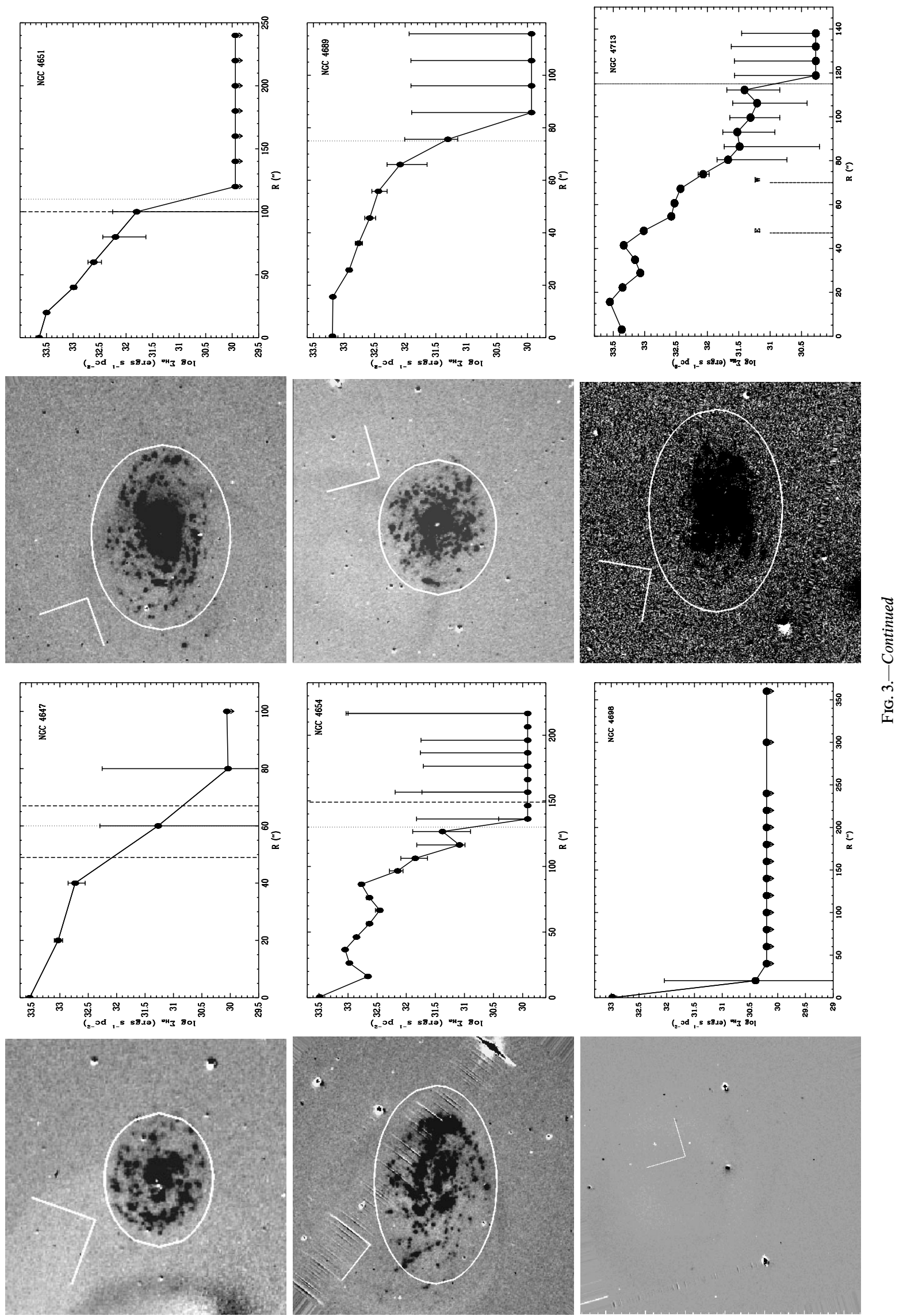

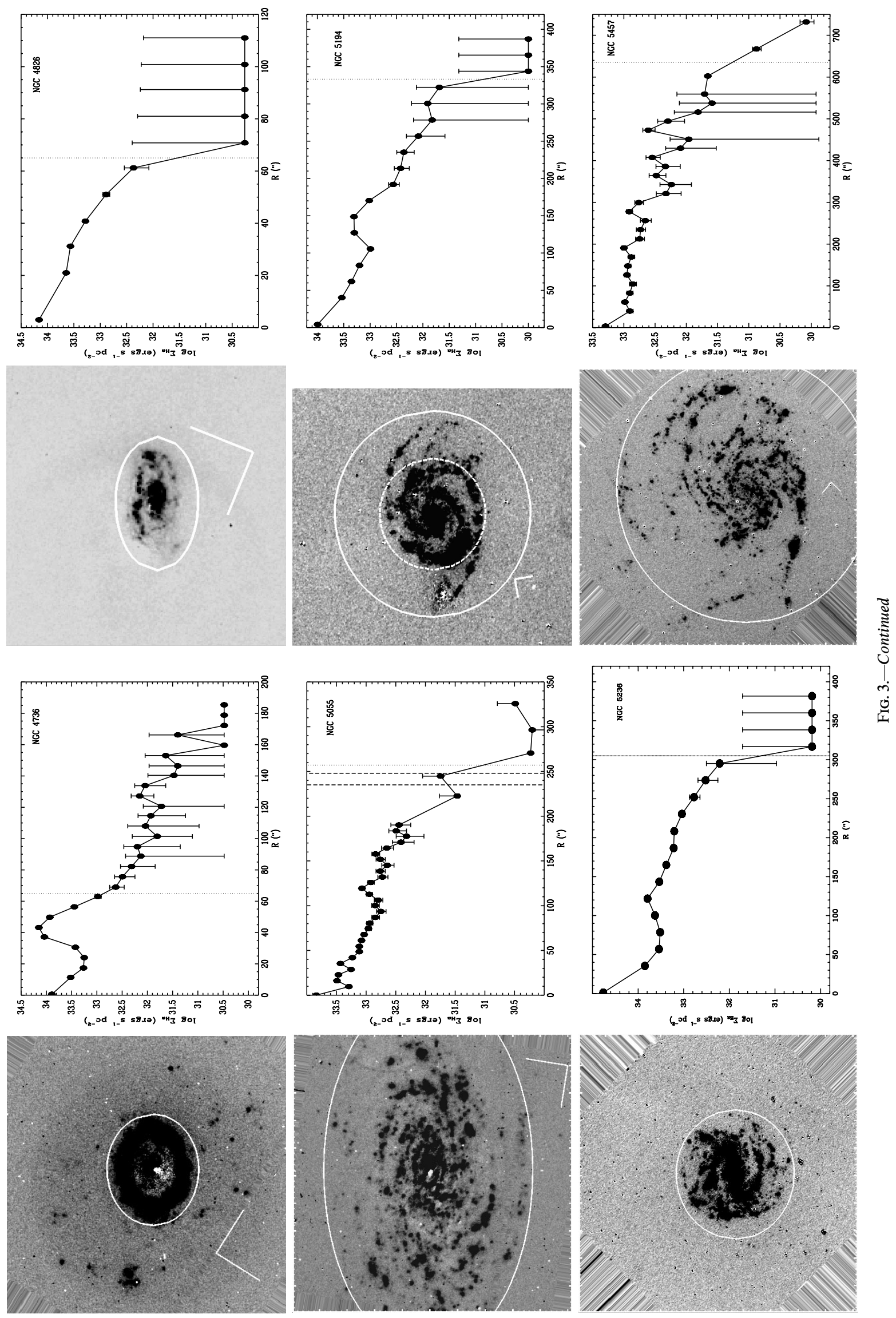

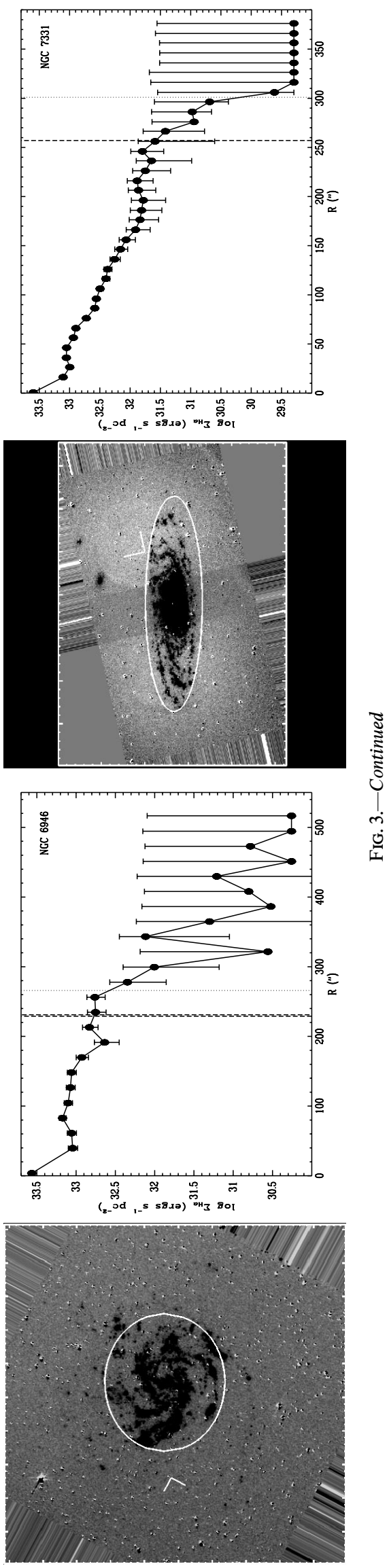
molecular gas disk is truncated abruptly at $R \approx 250^{\prime \prime}$, then the value of $\alpha\left(R_{\mathrm{H}}\right)$ could be as low as 0.25 . This scenario is unlikely as it would produce a strong discontinuity in the total gas surface density. However, fitting the exponential profile to only the less sensitive data $\left(R_{\mathrm{CO}}=150^{\prime \prime}\right.$ instead of $R_{\mathrm{CO}}=250^{\prime \prime}$ ) yields a flatter gradient in the molecular gas density and overestimates the threshold gas density by $\sim 40 \%$. This type of systematic error appears to dominate the systematic error in $\alpha\left(R_{\mathrm{H}}\right)$ for a number of galaxies in our sample.

\subsection{Threshold Radii}

Figure 3 shows $\mathrm{H} \alpha+[\mathrm{N}$ II $]$ images and radial surface brightness profiles for the entire sample. The threshold radii are marked. Star formation thresholds, as described for NGC 5236, are easily identified in 27 of the 32 galaxies in the sample. In most of these galaxies the drop in SFR per unit area at the threshold is very sharp, declining by a factor of 3-400 over a single $10^{\prime \prime}$ resolution element. The change in star formation efficiency, i.e., SFR per unit gas mass, is similar. The profiles for NGC 2903, NGC 4535, and NGC 4736 do not show such strong discontinuities, but the sharp change in profile slope in the outer disks does seem to reflect some type of threshold in the star formation law. The $\mathrm{H} \alpha$ surface brightness in NGC 2841 and NGC 4698 is too low to determine whether the threshold effect is present.

In many disks, $\mathrm{H}$ II regions are seen beyond the threshold radius (Ferguson et al. 1998; Lelievre \& Roy 2000). Their covering factor is much lower than it is within the main star-forming disk $\left(R<R_{\mathrm{H}}\right)$. It seems plausible that the local $\mu_{\text {gas }} / \mu_{\text {crit }}$ ratio is higher in these regions. In NGC 2903, for example, the $\mathrm{H}$ in regions beyond the threshold radius are clearly associated with density perturbations caused by the spiral arms. The closer the mean gas density is to the critical density, the more frequently we expect a given region to experience a perturbation sufficient to bring it above the threshold.

\subsection{Subcritical Disks}

Our sample contains seven subcritical disks where $\alpha$ never reaches the empirically defined threshold value: $\alpha_{Q}=$ 0.63 in K89 and $\alpha_{Q}=0.69$ in a subsequent section of this paper. One member of our sample with a subcritical disk, NGC 2403, shows widespread star formation activity, and this failure of the Toomre criterion will be discussed later. Figure 4 shows the instability profiles for the other six galaxies with subcritical disks. The gas density becomes a progressively larger fraction of the critical density [at the radius of maximum $\alpha(R)]$ in this order: NGC 4698, NGC 4639, NGC 3031, NGC 2841, NGC 4548, and NGC 4571.

To facilitate comparison of their $\mathrm{H}$ II region distributions, the six galaxies are displayed with the same intensity limits in Figure 3. Starting with the most stable disk in the sample, NGC 4698, only a few, small $\mathrm{H}$ in regions are detected. The $\mathrm{H}$ II regions in NGC 3031 are confined to the spiral arms whose extent may be set by an outer Linblad resonance (see Adler \& Westpfahl 1996; but see also Westpfahl 1998). The distribution of $\mathrm{H}$ II regions is much more widespread in NGC 2841, consistent with an instability that approaches the critical value. In NGC 4548, the annulus extending from about $0.5 R_{\mathrm{H} \text { I }}$ to $R_{\mathrm{H} \text { II }}$ is near the instability limit, and a pair of spiral arms is present there. The bar in NGC 4548 and ram pressure stripping have strongly influenced the gas distribution (Vollmer et al. 1999) and consequently the star

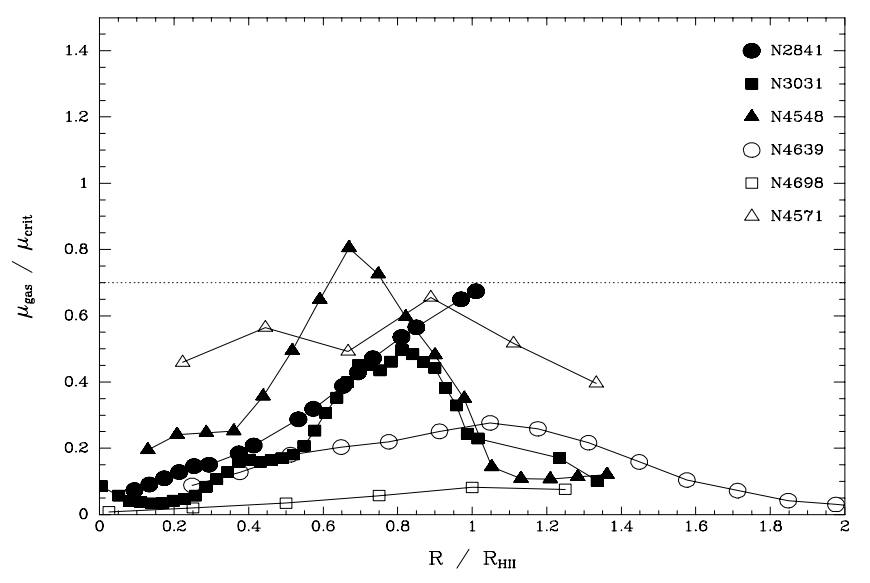

FIG. 4.-Radial variation of the instability parameter in subcritical disks. If no threshold radius was found, the radius of maximum instability was used to normalize the abscissa.

formation. This comparison indicates that (1) external perturbations have an important impact on where star formation occurs in subcritical disks and (2) $\mathrm{H}$ II regions become more prevalent as the instability parameter increases.

The paucity of star formation in these six galaxies cannot be attributed to low gas surface density. The disk of NGC 4698 is a special case in as much as the gas was probably removed in a collision with another galaxy (Valluri \& Jog 1990). The gas surface density in three of the other five subcritical disks exceeds the median threshold density (see $\S 3.4$ and Fig. 5). This result has implications beyond early-type spiral galaxies. Low surface brightness galaxies
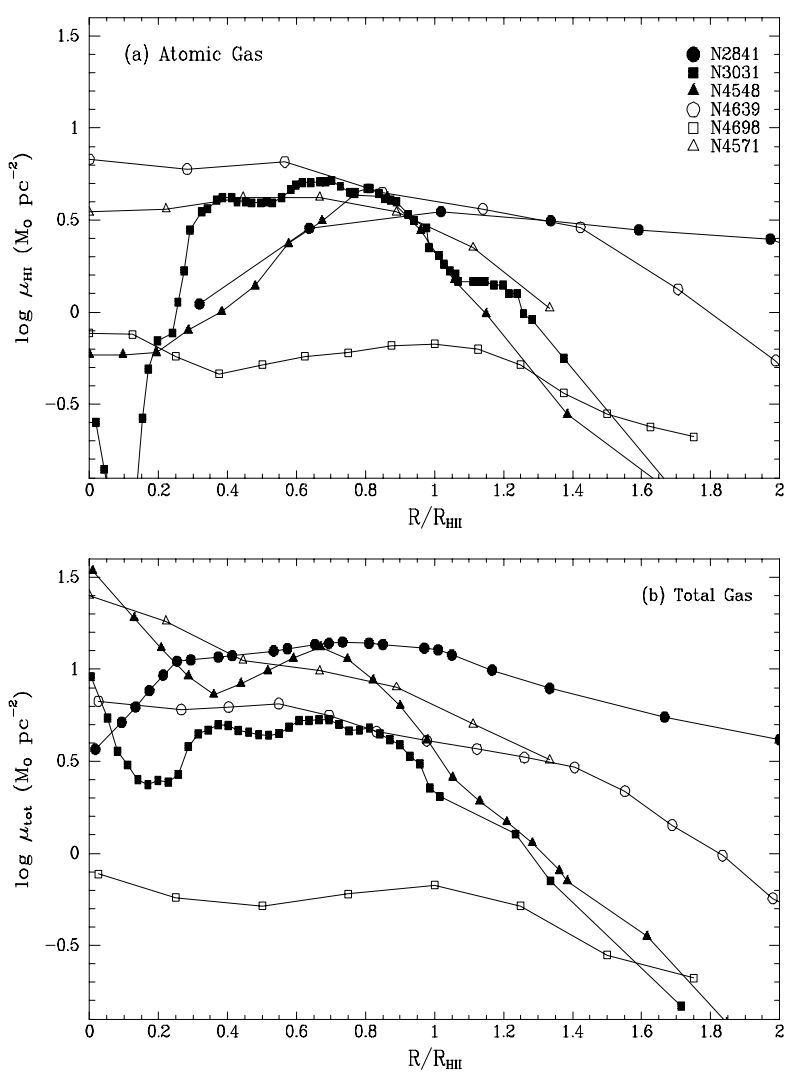

FIG. 5.-Gas surface density in subcritical disks 
also present a sparse distribution of $\mathrm{H}$ II regions and subcritical gas densities (van der Hulst et al. 1993), but the paucity of $\mathrm{H}$ II regions can be attributed to the low gas surface density (only a few solar masses per square parsec). Our analysis strongly suggests that it is the ratio $\mu_{\text {gas }} / \mu_{\text {crit }}$ rather than the gas surface density alone that sets the threshold for widespread star formation.

\subsection{The Relative Fraction of Atomic and Molecular Gas}

Using the data described in $\S 2$, we measured the surface density of atomic and molecular gas at $R_{\mathrm{H} \text { II }}$ in each galaxy. Figure 6 shows that the gas surface density ranges from 0.7 to $40 M_{\odot} \mathrm{pc}^{-2}$ at the outer threshold. In a few galaxies with prominent gas rings, like NGC 4402 and NGC 5055, the apparent edge of the molecular gas disk does appear to be associated with the edge of the star-forming disk. However, in half of the galaxies in our sample, atomic gas contributes most of the gas surface density at $R_{\mathrm{H}}$. In eight galaxies, the molecular gas surface density is essentially zero at $R_{\mathrm{H}_{\text {II }}}$ for any reasonable extrapolation of the $\mathrm{CO}$ intensity profile, and threshold radii predicted from the molecular gas density alone would grossly underestimate the observed threshold radii. (The individual star-forming regions almost certainly contain unresolved molecular clouds, but their contribution to the gas surface density is apparently negligible in these regions.) Any viable threshold mechanism must explain the wide range in $\mu_{\text {gas }}\left(R_{\mathrm{H}}\right)$ and not depend on whether the gas is in atomic or molecular form. The gravitational stability model meets both of these criteria. We emphasize that it predicts the formation of large, selfgravitating clouds, not stars. The good agreement with the observed radii of the star-forming disks, however, strongly suggests that star formation will happen once the clouds form.

At the median threshold gas density the atomic gas fraction ranges from $20 \%$ to $100 \%$. Yet at densities twice as high, the interstellar medium (ISM) is almost entirely molecular. The gas is almost entirely atomic at surface densities that are half the mean. Much of the gas mass is in molecular form in disks with high gas surface densities, including the subcritical disk of NGC 2841. The high atomic gas fractions in the other subcritical disks are consistent with their average to low gas density. This division in atomic gas fraction is expected if the column of $\mathrm{H}$ I required

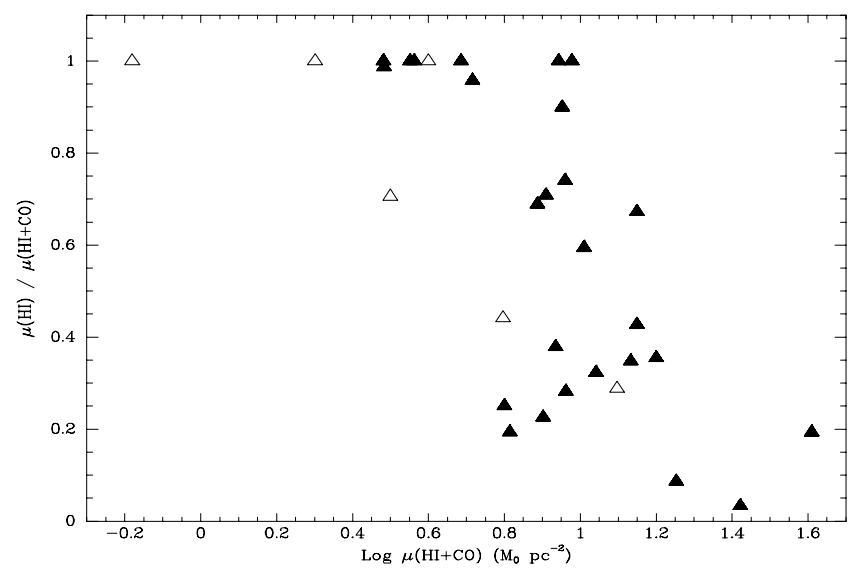

Fig. 6.-Mass fraction of atomic gas vs. total gas mass at the star formation thresholds. The subcritical disks are represented by open triangles. to shield the molecular gas from radiation (that would dissociate it) is fairly uniform among disk galaxies. The implied threshold column for molecular gas formation ranges from 5 to $15 M_{\odot} \mathrm{pc}^{-2}$.

It is interesting to examine whether the disks that are mostly molecular at $R_{\mathrm{H} \text { II }}$ become dominated by $\mathrm{H}$ I where the gas surface density drops into the $5-15 M_{\odot} \mathrm{pc}^{-2}$ range. Generally, we find that no $\mathrm{CO}$ emission is detected at this radius, so the data for the outer disks are consistent with the same $\mathrm{H}_{2} / \mathrm{H}$ I self-shielding column density. The only disk that clearly has a low atomic gas fraction at low total gas column is NGC 4579.

\subsection{The Instability Parameter at the Outer Threshold}

Critical surface densities for instability were computed from the rotation curves and compared to the gas densities described in the previous section. The median value of the instability parameter at the outer thresholds, $\alpha_{Q}=0.69$, was found to be consistent with the K89 value. To reveal the physical limitations of this model, we examine the stability of the disks at the threshold radii in greater detail than previous work. Three types of second-order effects are examined: (1) Does the scatter in the $\alpha\left(R_{\mathrm{H} \text { II }}\right)$ values reflect the accuracy of the model or the magnitude of systematic errors? (2) Can the sharpness of the threshold be predicted from the stability properties of the disk? and (3) Is the stability of the gas disk influenced by the stellar disk?

\subsubsection{Disk Asymmetry}

The epicyclic frequency and, consequently, the critical density have significant errors where the rotation curve is poorly determined as in highly inclined disks, disks with kinematic disturbances near $R_{\mathrm{H} \text { II }}$, and disks with offset dynamical and photometric centers. Within our sample, the projection effects are largest for NGC 628 where a $1^{\circ}$ uncertainty in the disk inclination implies a $20 \%$ uncertainty in $\mu_{\text {crit }}$. The rotation curves of NGC 5457 and NGC 5194 are not well defined at $R_{\mathrm{H} \text { II }}$ because of tidal disturbances, so the threshold instability parameter is not defined in these two galaxies. The dynamical center of NGC 925 is not coincident with the photometric center (in $\mathrm{H} \alpha$, continuum, or $\mathrm{H} \mathrm{I}$ ), a situation common in barred Magellanic irregulars (Pisano, Wilcots, \& Elmegreen 2000 and references therein). In general, though, uncertainty about $\mu_{\text {crit }}$ is small compared to the error in the $\mu_{\text {gas }}$ measurement.

The largest systematic errors in the measurement of $\mu_{\mathrm{gas}}$ are introduced by the asymmetry of the star-forming disks, i.e., the lack of axial symmetry or roundness. To quantify the impact on the threshold measurements, we measured a threshold radius and stability profile along the major axis of each galaxy. Comparison to the azimuthally averaged quantities reveals the magnitude of the azimuthal variations in the instability parameter. The largest variations are illustrated in Figure 7. We find the following correlations between the instability parameter and the distribution of star formation in these disks:

1. Recent star formation activity in NGC 4254 is skewed toward the northeastern half of the disk. The threshold radius on the western side is only $60 \%$ of its average value. The instability parameter along the eastern side of the major axis is also higher than it is on the western side. The threshold radius and stability parameter derived from the azimuthally averaged profile are heavily weighted by the eastern side of the disk. 

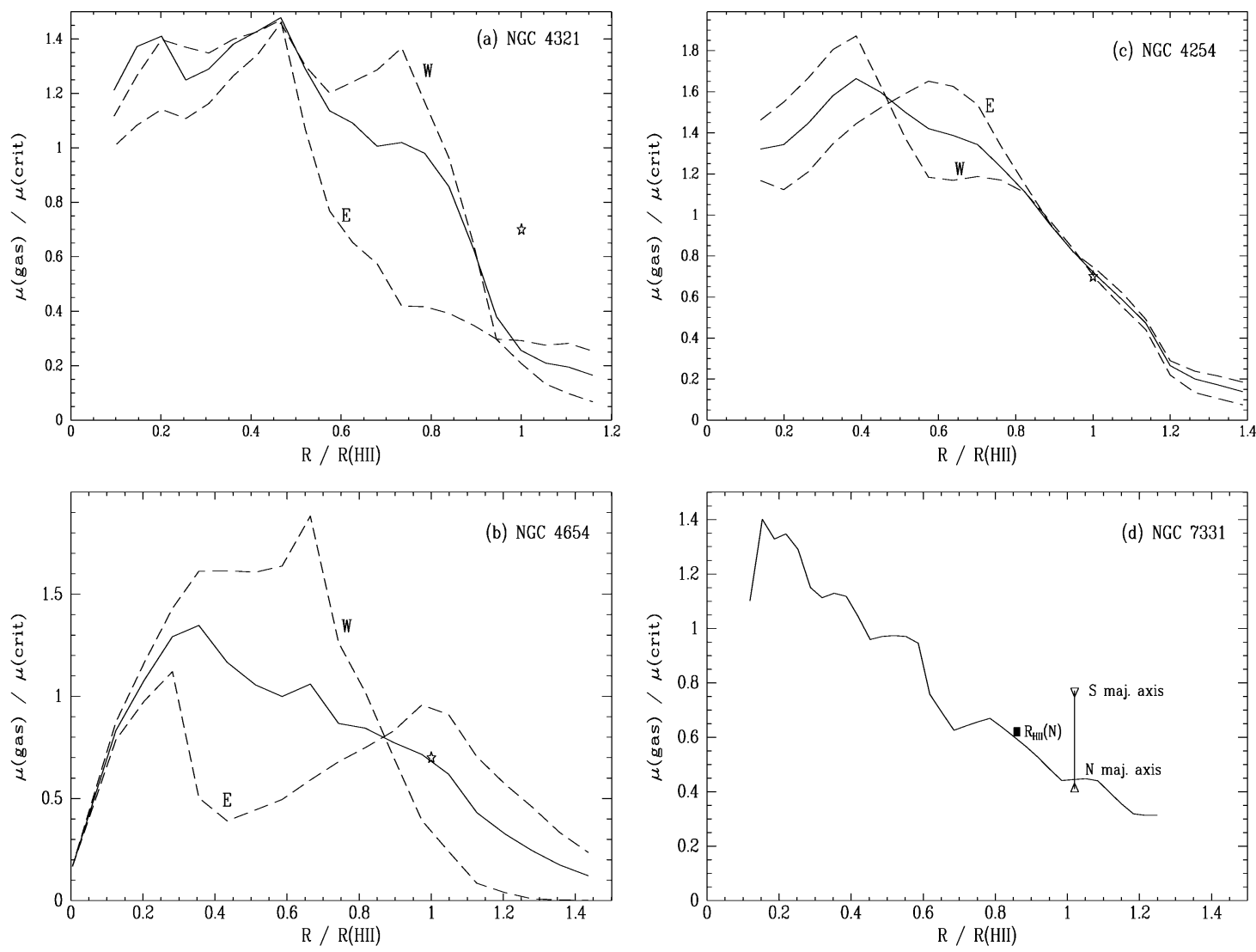

Fig. 7.-Azimuthal variation in the instability parameter. The solid lines represent an azimuthal average. The dashed lines are cuts along the major axis. Note that in (b) the $\mathrm{H}$ I surface density was measured at position angles of $\pm 90^{\circ}$, and the molecular gas was measured along the major axis, which is $60^{\circ}$ off this H I strip.

2. In NGC 4321, the average threshold radius $R_{\mathrm{H} \text { п }}$ provides a poor description of the irregular outer, $\mathrm{H} \alpha$ isophotes. At a position angle of $90^{\circ}$, for example, the $\mathrm{H}$ II regions extend to a radius of $0.9 R_{\mathrm{H} \text { II }}$ to the west but only $0.6 R_{\mathrm{H}}$ to the east. Inspection of Figure 3 shows that the two-arm spiral pattern also loses its definition at these radii. The surface density is $\sim 0.7 \mu_{\text {crit }}$ at the threshold along either direction of this cut because the gas density declines much more steeply with radius on the east side of the galaxy. In contrast, when the gas densities from each side of this disk are averaged together, the value of the instability parameter at $R_{\mathrm{H} \text { II }}$ is underestimated by a factor of 2 . Better spatial coverage drives the threshold stability parameter toward $\alpha\left(R_{\mathrm{H}}\right)=0.7$.

3. The star formation activity in NGC 4654 extends farthest to the southeast, but the $\mathrm{H}$ iI regions are faint and sparse here. The $\mathrm{H} \alpha$ surface brightness is much higher on the western side of NGC 4654 where the star formation threshold is much sharper. The gas surface density is subcritical over much of the eastern side of the galaxy and supercritical on the western side, consistent with the paucity of $\mathrm{H}$ II regions to the east. On the eastern side, the instability parameter decreases more slowly with radius, however, explaining the larger radial extent of the $\mathrm{H}$ II regions. The sharp gradient in the instability parameter on the western side is likely not resolved, so the value of the instability parameter, $\alpha=1.6$, at the western threshold is overestimated.

4. In NGC 7331, the star formation activity is skewed toward the southern half of the disk. The azimuthally aver- aged $\mathrm{H} \alpha$ profile is weighted toward this side of the disk, so the southern edge of the star-forming disk defines $R_{\mathrm{H}}$. Along the southern side of the major axis, the gas density is $0.8 \mu_{\text {crit }}$ at $R_{\mathrm{H} \text { I }}$. At this radius, $\alpha=0.4$ on the northern side of the disk. However, at the smaller radius defined by the northern edge to the star-forming disk, the gas density is $0.6 \mu_{\text {crit }}$. In this case the azimuthally averaged value of the instability parameter, $\alpha\left(R_{\mathrm{HII}}\right)=0.4$, clearly underestimates the true value at the edge of the star-forming disk.

We have demonstrated that using azimuthally averaged gas densities and SFRs can lead to errors in $\alpha\left(R_{\mathrm{H}}\right)$ as large as a factor of 2 when the disk is highly nonaxisymmetric. The theoretical basis for the Toomre stability criterion breaks down in these irregular systems, so it is noteworthy that a local $Q$ criterion appears to be so useful in describing the distribution of star formation in these highly disturbed disks. The examples presented demonstrate that the distribution of star-forming regions clearly becomes much more sparse, similar to the subcritical disks, in regions where the instability parameter is low. The threshold values of the instability parameter also show a tendency to converge when better spatial coverage is used.

\subsubsection{Star Formation beyond the Threshold Radius}

Gravitational threshold models only predict a sharp edge to the star-forming disk if the gas disk is perfectly smooth. The structure of the ISM in real galaxies must modify this picture (K89). Nonaxisymmetric modes and local perturbations generate structure on large and small scales, respec- 
tively. Such regions may allow cloud formation (and subsequently star formation) even where the azimuthally averaged gas density is subcritical. For example, in $\S 3.3$, the spiral density waves in the subcritical disks of NGC 3031 and NGC 4548 are supported where the gas surface density exceeds $0.3 \mu_{\text {crit }}$ rather than $0.69 \mu_{\text {crit }}$. The lower threshold is consistent with models of swing amplification. The more isolated $\mathrm{H}$ II regions in the subcritical disks are generated by a different mechanism. We suggest that random density perturbations generate some supercritical regions in disks that have surface densities close to the critical density for instability. Support for this viewpoint comes from the subcritical disks. Isolated $\mathrm{H}$ II regions were found most frequently when the gas surface density was only slightly subcritical, and no $\mathrm{H}$ II regions were found where the gas surface density was less than $10 \%$ of the critical density. As the average gas surface density approaches the critical density, the amount of star formation activity in isolated, starforming regions clearly increases. Could a combination of local perturbations and spiral arms also explain the existence of $\mathrm{H}$ in regions at radii beyond the threshold radius? If so, star formation activity should be more prevalent where the $\mu_{\text {gas }} / \mu_{\text {crit }}$ ratio remains high (i.e., greater than 0.3 ) beyond $R_{\mathrm{H} \text { II }}$.

To test this hypothesis, we need to minimize the systematic errors caused by disk asymmetry at $R_{\mathrm{H} \text { II }}$, so we compare the 11 galaxies with the smallest azimuthal variations in threshold radius. Figure 8 shows the radial stability profiles for 10 members of our sample; the profile for NGC 5236 was shown previously. The spread in the instability parameter is only a factor of 2 at the threshold radius where the profiles converge. Beyond the threshold radius, the shallowest gradient is found in the disk of NGC 2403, which remains marginally stable. Not surprisingly, many H II regions are seen beyond $R_{\mathrm{H}}$ in NGC 2403. The gas surface density in NGC 6946 also remains close to the critical density, i.e., $\mu_{\text {gas }} \geq 0.4 \mu_{\text {crit }}$, out to $2 R_{\mathrm{H} \mathrm{II}}$, and a large number of $\mathrm{H}$ II regions are seen beyond $R_{\mathrm{H} \mathrm{II}}$ (see Ferguson et al. 1998). In contrast, the steepest gradients in the instability parameter,

$$
\left|\frac{d\left(\mu_{\text {gas }} / \mu_{\text {crit }}\right)}{d R}\right|>0.1 \mathrm{kpc}^{-1}
$$

are found in NGC 4402, NGC 4713, and NGC 5236. None of these galaxies show much $\mathrm{H} \alpha$ emission beyond the threshold radius. The instability parameter is also low outside the threshold radius in NGC 2903, but the star formation is confined to the spiral arms locally. The likelihood of finding isolated $\mathrm{H}$ II regions outside $R_{\mathrm{H} \text { II }}$ therefore appears to scale with the value of the instability parameter.

It is interesting to examine why, beyond $R_{\mathrm{H} \mathrm{II}}, \alpha(R)$ remains high in some disks and drops sharply in others. Figure 9 shows the gas surface density profiles for the same 10 galaxies. The $\mathrm{H}$ I disks of the Virgo Cluster galaxies NGC 4394, NGC 4402, and NGC 4689 appear to have been stripped at radii $R \geq R_{\mathrm{H}}$. When the molecular gas density is included, the difference in total gas surface density relative to the field galaxies is reduced, but the Virgo members still tend to have lower values of the instability parameter outside $R_{\mathrm{H} \text { Ir }}$. Inspection of Figure 3 shows that the Virgo galaxies show $\mathrm{H}$ II regions beyond $R_{\mathrm{H} \text { II }}$ less frequently than the field sample.

\subsubsection{Variations in the Threshold Stability Parameter with Galaxy Type}

Figure 10 shows our best estimates of $\alpha\left(R_{\mathrm{H}}\right)$ at the outer edges of 26 disks. $^{3}$ The significance of the weak trend with galaxy type is difficult to assess quantitatively. The error bars represent limiting values and are set by the magnitude of systematic errors associated with one-dimensional gas profiles in nonaxisymmetric disks, tidal disturbances, the

${ }^{3}$ The six galaxies with subcritical disks are omitted. NGC 5194 and NGC 5457 are not shown since the rotation curves are not well defined at $R_{\mathrm{H} \text { II }}$.

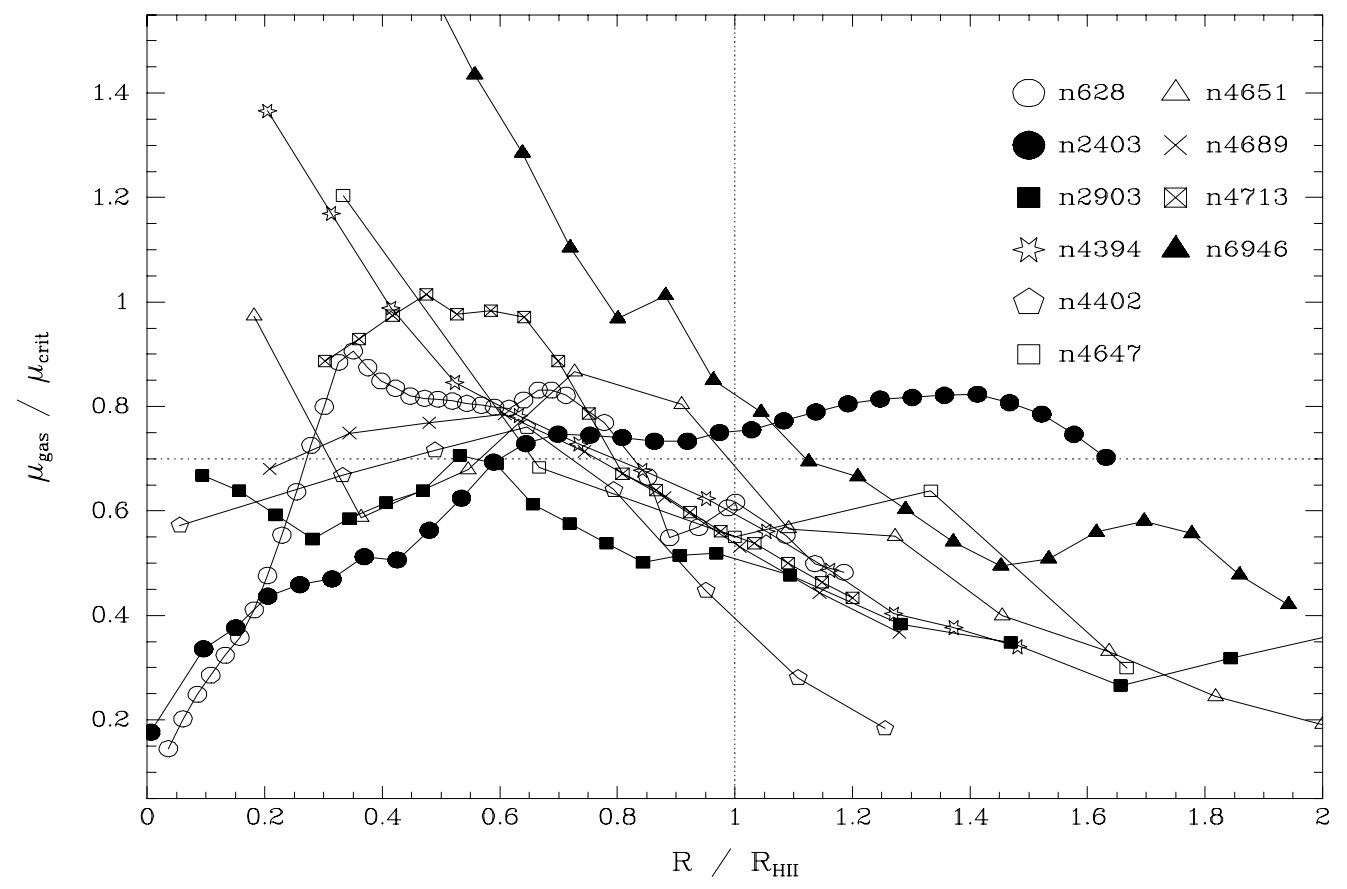

FIG. 8.- Radial variation in the instability parameter. The disks with the least azimuthal variation in threshold radius are shown. The galactocentric radius is normalized to the threshold radius. Dotted lines mark the mean ratio of the gas surface density to the critical density at the threshold radius. 

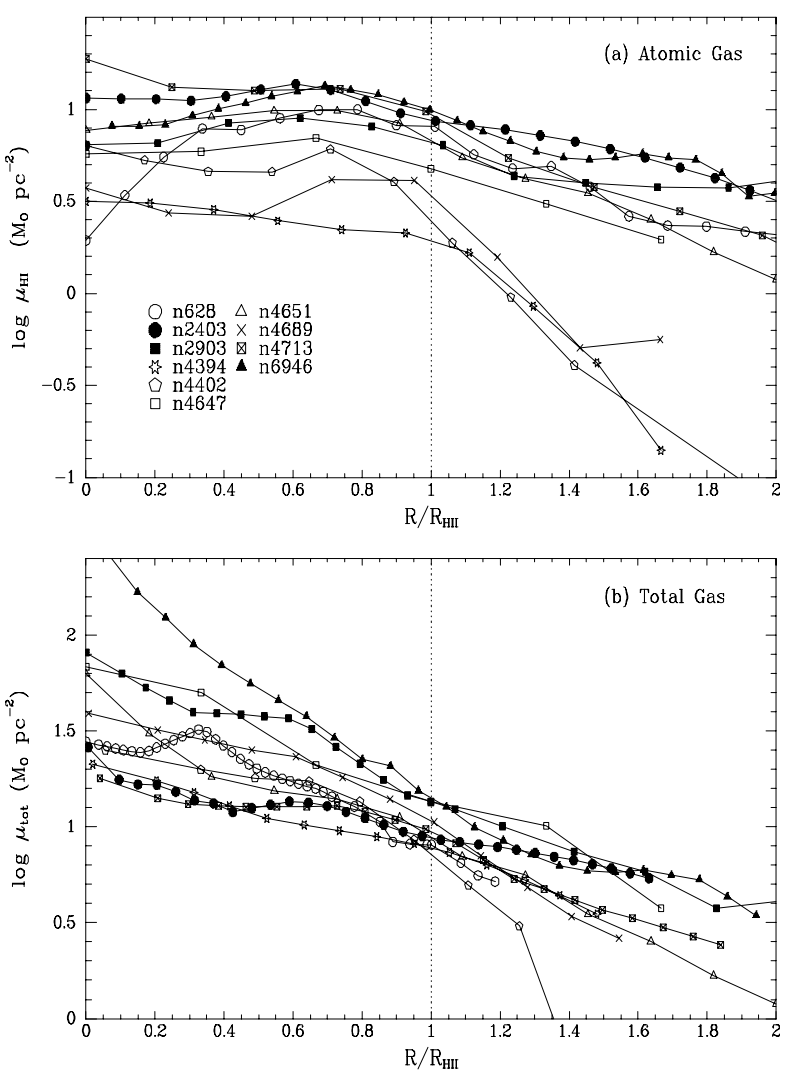

FIG. 9.-Radial variation in gas surface density. The disks with the least azimuthal variation in threshold radius are shown.

extrapolation of the $\mathrm{CO}$ intensity profile to larger radii, and disk inclination. The dominant error terms differ in the early-type and late-type disks, so we discuss their $\alpha\left(R_{\mathrm{H} \text { II }}\right)$ values separately.

The earlier type disks ( $\mathrm{Sa}-\mathrm{Sb})$ in Figure 10 tend to have $\alpha\left(R_{\mathrm{H} \mathrm{II}}\right)$ values lower than the sample median. The discrep-

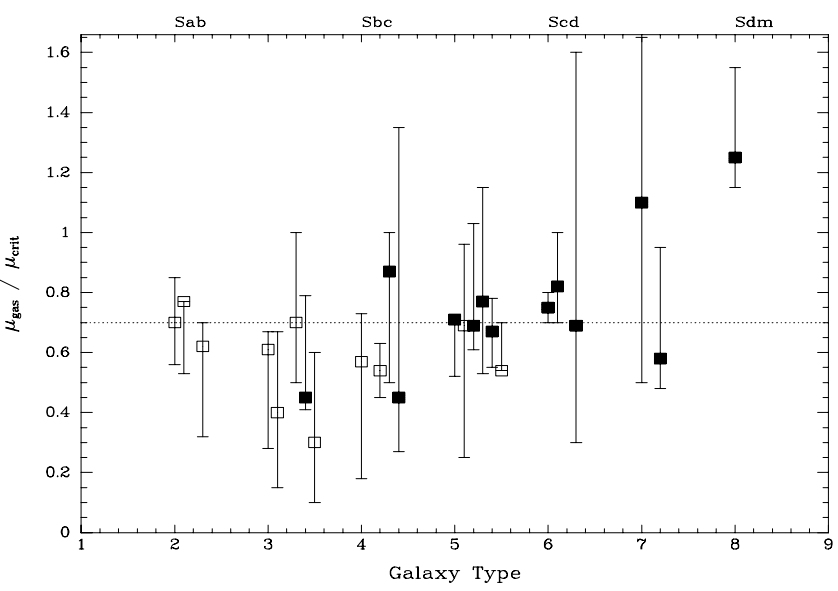

FIG. 10.- Instability parameter at the star formation threshold vs. revised morphological type ( $\mathrm{RC} 2 \mathrm{~T}$ type) of the galaxy. (Note that galaxy type is an integer quantity, and the decimals are used merely to separate points.) Filled (open) symbols represent surface densities dominated by atomic (molecular) gas, respectively. From left to right, the points represent these galaxies: NGC 4736 (2.0), NGC 4826 (2.1), NGC 4569 (2.3), NGC 4394 (3.0), NGC 4402 (3.1), NGC 4501 (3.3), NGC 7331 (3.4), NGC 4579 (3.5), NGC 4689 (4.0), NGC 2903 (4.2), NGC 5055 (4.3), NGC 4321 (4.4), NGC 4651 (5.0), NGC 5236 (5.1), NGC 628 (5.2), NGC 4254 (5.3), NGC 4535 (5.4), NGC 4647 (5.5), NGC 2403 (6.0), NGC 6946 (6.1), NGC 4654 (6.3), NGC 925 (7.0), NGC 4713 (7.2), and NGC 4178 (8.0). ancy is not significant for NGC 2903, NGC 4321, and NGC 7731. The range of $\alpha$ values shown for each of them is consistent with the sample median, 0.69 , because corrections for nonaxisymmetric structures were shown to drive $\alpha$ in this direction (S 3.5.1). The $\alpha\left(R_{\mathrm{H}}\right)$ values for the earlytype galaxies NGC 4394, NGC 4402, NGC 4579, and NGC 4689 range from 0.5 to 0.6 and depend on extrapolations of the $\mathrm{CO}$ intensity profile. In all of the galaxies where molecular gas dominates the gas density at the threshold, $\alpha$ is less than or equal to 0.69 , and most of these galaxies are type $\mathrm{Sab}-\mathrm{Sbc}$. It is unlikely that the gas surface density is systematically underestimated in these disks because the two largest biases in interpretation of the $\mathrm{CO}$ measurements work to increase the molecular gas density. First, since the $\mathrm{CO}$ emission is not well resolved, particularly in NGC 4394, NGC 4402, and NGC 4689, the tendency is to overestimate scale lengths and artificially raise the molecular gas surface density at the threshold radii. Second, the $\mathrm{CO} / \mathrm{H}_{2}$ conversion factor is expected to be lower in disks with low metallicity. If the early-type spirals are more evolved than the late-type spirals, i.e., they have consumed a larger fraction of their original gas content, then they should have higher metallicity on average. Hence, we have no reason to expect a low $\mathrm{CO} / \mathrm{H}_{2}$ conversion factor in the early-type galaxies. It is plausible then that the $\mu_{\text {gas }} / \mu_{\text {crit }}\left(R_{\mathrm{H} \text { II }}\right)$ ratios are somewhat lower than the mean in these early-type disks.

In contrast, the threshold values of the instability parameter for the $\mathrm{H}$ I-dominated edges scatter to both sides of 0.69 . Lower mean metallicities in the later type disks would introduce an artificial negative slope in Figure 10, which is not seen. We find no trend toward higher values of the instability parameter in late-type galaxies. In NGC 628, the estimated threshold value of the instability parameter, $\alpha\left(R_{\mathrm{H}}\right)=0.86$, is consistent with the mean, $\alpha_{Q}=0.69$, given the uncertainty in the rotation speed described in $\S 3.5 .1$. In NGC 925, the instability parameter is overestimated on the western side of the galaxy when the offset between the photometric and dynamical centers of this galaxy is neglected. (The kinematic radius is smaller than the $\mathrm{H} \alpha$ radius, so $\kappa$ is higher than we estimated assuming common photometric/dynamic centers.) A detailed analysis using $\sigma_{g}=10 \mathrm{~km} \mathrm{~s}^{-1}$ found that $\alpha$ is 0.3 at the threshold (Pisano et al. 2000), which corresponds to $\alpha=0.5$ for our assumed value of $\sigma=6 \mathrm{~km} \mathrm{~s}^{-1}$. Evaluation of the instability is particularly difficult in these late-type spirals, owing to the nonaxisymmetric structures. We find no trend toward lower $\alpha\left(R_{\mathrm{H}}\right)$ in the low angular velocity galaxies in our sample, all of type Scd to the Sdm, as one might have expected based on the low $\alpha$ values reported in dwarf irregular galaxies (HEB98).

\subsubsection{Conclusions}

The asymmetries of many disks in the sample compromise the accuracy of the stability analysis. We have demonstrated, however, that when disks are examined along position angle cuts that eliminate this uncertainty, the convergence of the stability profiles at the threshold radii is tighter. Outside the threshold radius, the disks with gas densities well below the critical density do not show many $\mathrm{H}$ II regions while those with prominent populations of $\mathrm{H}$ II regions tend to be near the threshold surface density. The weak trend toward lower values of $\alpha_{Q}$ in earlier type spiral galaxies is consistent with these disks having a larger stellar mass fraction (e.g., Jog \& Solomon 1984a, 1984b). 


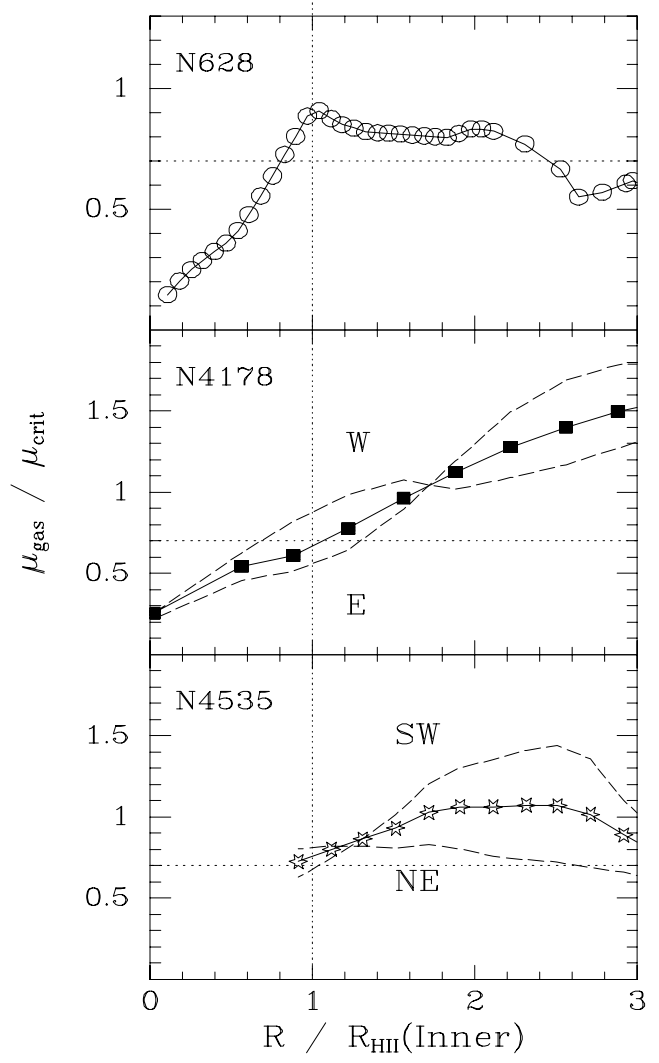

FIG. 11.- Instability parameter normalized to the location of the threshold in the inner disk. The dotted lines show the median value of the instability parameter at the outer thresholds for reference.

\subsection{Inner Disk Thresholds}

We also examined whether simple gravitational stability arguments might be as successful describing star formation thresholds in the inner regions of disks as we have shown them to be at the outer edge of star-forming disks.

The sharpest star formation thresholds in the inner disk are associated with rings of intense star formation activity (e.g., see NGC 4569, NGC 4579, NGC 4639, and NGC 4736 in Fig. 3). Does the instability parameter, $\alpha(r)$, increase across these rings? The beamwidth of the $\mathrm{H}$ I and $\mathrm{CO}$ observations is inadequate to evaluate the instability parameter across the rings in NGC 4569 and NGC 4639. The major axis instability profile across NGC 4579 reaches $\alpha=0.6$ on the western side of the ring, but the gas density along the eastern major axis is much lower than the critical density. The ring of $\mathrm{H}$ II regions in NGC 4579 is brighter on the west side than the east side, so star formation in the ring appears to be correlated with higher values of the instability parameter, i.e., lower $Q$ values. In NGC 4736, the ring of $\mathrm{H}$ II regions at $R \sim 45^{\prime \prime}$ coincides with an inner Lindblad resonance (Gerin, Casoli, \& Combes 1991; Gu et al. 1996; Wong \& Blitz 2000). The spatial resolution of the gas data does not resolve the 680 pc wide ring, but the lower limits on the gas surface density in the ring are sufficient to raise the instability parameter to values of $0.6-1.0$ near the inner edge of the ring $\left(R \approx 34^{\prime \prime}\right)$. Higher resolution data indicate that $Q<1$ in localized regions of the ring and $Q \sim 3$ averaged across the ring (Wong \& Blitz 2000).

A few galaxies without rings have gas surface densities less than $0.69 \mu_{\text {crit }}$ in their inner disks. We show the $\alpha(R)$ profiles for the three with the best spatial resolution in
Figure 11. We expect to see little $\mathrm{H} \alpha$ emission at radii less than the inner threshold radii in these galaxies. In Figure 3, the disks of NGC 628 and NGC 4535 show little star formation inside the predicted threshold radii of $70^{\prime \prime}$ and $50^{\prime \prime}$, respectively. Star formation in the central region of NGC 4178 is confined to the bar, where the gas density is likely higher than the azimuthal average, so the star-forming regions may not be subcritical. The inner thresholds in these three galaxies are therefore consistent with the gravitational threshold model. Only the star formation in the central region of NGC 2403 is in direct conflict with the gravitational threshold model.

The inner regions of many spiral galaxies have a deficit of $\mathrm{H} \alpha$ emission, relative to a fitted exponential disk (e.g., NGC 628, NGC 925, NGC 2403, NGC 3031, NGC 4321, NGC 4394, NGC 4535, and NGC 5236). Aside from the cases already discussed and NGC 4394, these breaks in the $\mathrm{H} \alpha$ surface brightness profile are not large enough to be called star formation thresholds. This property of the $\mathrm{H} \alpha$ surface brightness profiles was noticed by Hodge \& Kennicutt (1983), but the physical cause has not been established. The region of low SFR is clearly associated with a bar in NGC 5236, NGC 4394, and NGC 925. The inner threshold in NGC 3031 coincides with the inner Linblad resonance. Many galaxies with central $\mathrm{H} \alpha$ deficits have subcritical gas surface densities in the inner disk, and those with supercritical gas densities have bars or resonances. The only galaxy in this list with a supercritical gas density in the center and no identified bar or resonance is NGC 4321. Apparently, bars and resonances can inhibit star formation in the central regions of disks that are unstable to axisymmetric perturbations.

\section{DISCUSSION}

Our results support previous studies that have claimed that the outer edges of spiral disks are well described by the gravitational stability of a single-fluid, isothermal gas disk (K89). We have shown that this criterion is often a good description of star formation thresholds in the inner disk as well. The aim of this section is to gain further insight into the underlying physical processes that regulate disk star formation. We examine the galaxies where the gravitational instability model fails and evaluate the utility of a qualitatively different threshold condition. A clear prediction of the gravitational threshold scenario is that the stellar disks should affect $\alpha_{Q}$. We examine whether first-order accounting of the stellar disk changes the scatter in the distribution of $\alpha\left(R_{\mathrm{H}}\right)$ measurements. Finally, the impact of these star formation thresholds on the global SFR is summarized.

\subsection{Shear Models \\ 4.1.1. NGC 2403 and M33}

The gas density in the inner $240^{\prime \prime}$ of NGC 2403 is below the critical value and less than half the critical density within $150^{\prime \prime}$ of the center of the disk, yet the inner half of the disk is covered with $\mathrm{H}$ II regions. No other galaxy in our sample presents widespread star formation in regions where the gas disk is predicted to be stable. This contradiction and the very similar situation in M33 were noticed by K89. The most obvious explanations have been dismissed by Thornley \& Wilson (1995). In particular, if the $\mathrm{H}_{2} / \mathrm{CO}$ conversion factor were as much as 5 times higher than the Milky Way value, the implied increase in the molecular gas density would bring the total gas density up to the critical density in 
the inner disk without affecting the good agreement at the outer threshold. Comparison of virial and molecular masses for clouds in M33, however, indicates that the conversion factor is similar to the Galactic one (Wilson 1995). The metallicity and rotation speed of M33 are similar to NGC 2403, so Thornley \& Wilson (1995) find no reason to suspect a higher surface density of molecular gas in either galaxy.

The star-forming disks in many dwarf irregular galaxies also appear to be subcritical when the Toomre $Q$ parameter is used to estimate the critical density, and it has been suggested that the shear criterion (eq. [5]) describes the critical density better than the Toomre $Q$ criterion (Hunter \& Plummer 1996; HEB98). The disks of NGC 2403 and M33 share an important dynamical property with dwarf irregular galaxies. The shear rate is low in the inner disk where the rotation speed is rising slowly with radius. The disk stability analysis for the irregulars, while quite interesting, is not yet compelling. Large systematic uncertainties arise from the highly irregular shape of the disks, greater uncertainty about the $\mathrm{CO} / \mathrm{H}_{2}$ conversion factor at very low metallicities, and the assumption that the molecular/atomic gas fraction is constant with radius.

Figure 12 compares the shear criterion for the critical density to the Toomre critical density across NGC 2403 and M33. The gas surface densities for M33 data come from Newton (1980) and Young et al. (1995), and the threshold radius for star formation is $29^{\prime}$. In both spiral galaxies, the gas surface density exceeds $\mu_{\text {crit }}^{A}$ but falls below the Toomre critical density. The presence of widespread star formation
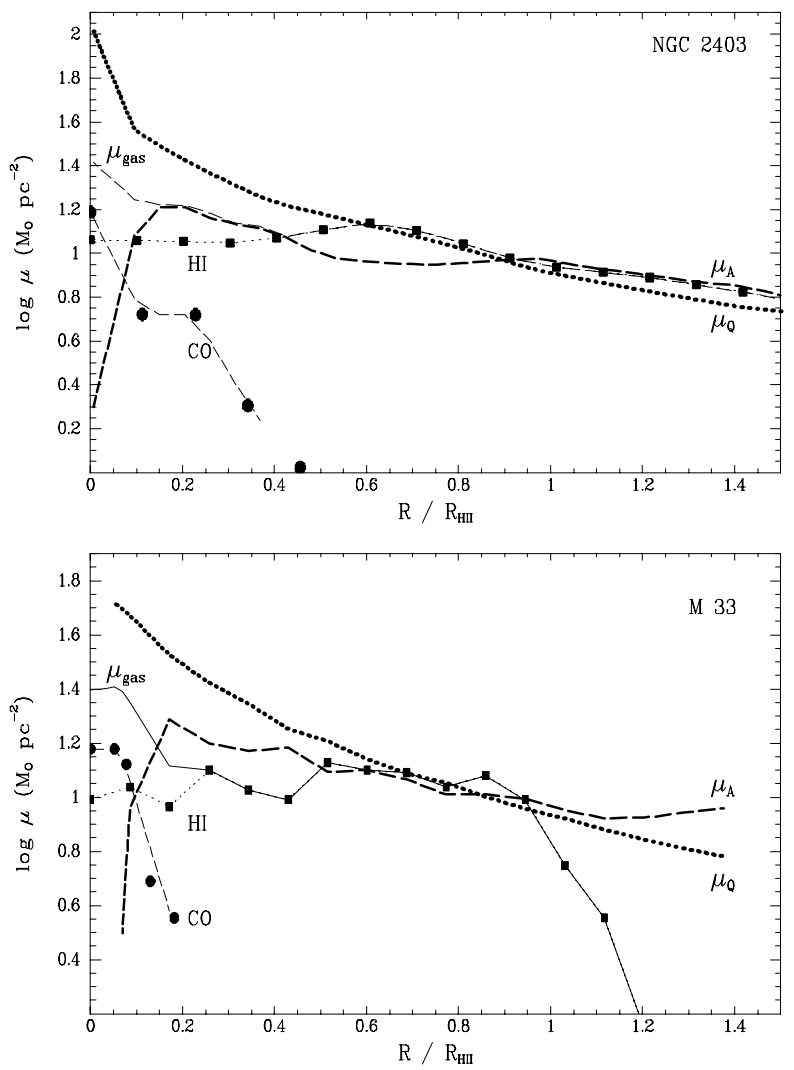

FIG. 12.-Comparison of the Toomre $Q$ and shear stability criteria in the inner disk. The observed gas surface density is shown by the solid line for comparison. The radial coordinate is normalized to the star formation threshold. can be interpreted as evidence that cloud formation is limited by the time available for cloud growth (in the presence of shear) rather than gravitational instability. It remains unclear whether this explanation is correct. One disk in our sample, NGC 4535, has an inner threshold that is well described by the $Q$ criterion but not the shear criterion. In addition, models of self-regulated star formation predict $Q \sim 1$ throughout the disk (Silk 1997). Departure from this equilibrium condition may indicate that the disk is in a transient state. The unusually high infall rate of neutral hydrogen onto the NGC 2403 disk, and perhaps M33 as well, supports this view (Schaap, Sancisi, \& Swaters 2000; Sancisi et al. 2000).

\subsubsection{Shear Criterion at the Outer Threshold}

The galaxies in our sample generally have flat rotation curves at the threshold radius, so the critical density based on the Toomre $Q$ criterion reduces to

$$
\mu_{\text {crit }}=\alpha_{Q} \frac{1.41 \sigma \Omega}{\pi G} .
$$

The shear criterion for the critical density has the same radial dependence where the rotation speed is constant,

$$
\mu_{\mathrm{crit} A}=\alpha_{A} \frac{0.5 \sigma \Omega}{\pi G} .
$$

Since the fitted value of $\alpha_{Q}=0.69$, we need $\alpha_{A}=2.0$ to obtain $\mu_{\text {gas }}=\mu_{\text {crit } A}$ at $R_{\mathrm{H} \mathrm{II}}$ for the disks with constant rotation speed at $R_{\mathrm{H} \mathrm{II}}$. This normalization factor is very close to that predicted by Elmegreen (Elmegreen 1993; HEB98). If the rotation speed is constant at the outer threshold, then no distinction can be made between these two criteria for the outer edge of a disk.

The galaxies in our sample with the largest gradients in rotation speed at $R_{\mathrm{H} \mathrm{II}}$ are NGC 4254, NGC 4394, NGC 4402, NGC 4535, and NGC 4569. The gradient in NGC 4394 is highly uncertain as a result of the low resolution of the $\mathrm{H}$ I position-velocity diagram for NGC 4394 . Figure 13 compares the Toomre (top panel) and shear (bottom panel) representations of the critical density for the other four galaxies. The scatter among the values of $\mu_{\text {gas }} / \mu_{\text {crit }}$ is actually smaller for the shear model than the Toomre $Q$ model. However, when the $\alpha_{A}$ term in the shear criterion is normalized using the galaxies with flat rotation curves at $R_{\mathrm{H} \mathrm{II}}$, as represented by NGC 2403 and M33 in Figure 13, the scatter in the $\mu_{\mathrm{gas}} / \mu_{\text {crit }}\left(R_{\mathrm{H} \text { II }}\right)$ values exceeds that obtained with the Toomre $Q$ criterion. The shear criterion does not appear to be as robust an indicator of the edges of star-forming disks in spiral galaxies as the Toomre criterion. The shear model was also unable to describe adequately the edges of the disks in dwarf irregular galaxies (HEB98).

\subsection{The Influence of Stellar Disks on Instability}

Real galaxies contain both gas and stars, and the system can be unstable even when both the stellar and gaseous components individually meet the requirements for stability (Jog \& Solomon 1984a, 1984b; Elmegreen 1995; Wang \& Silk 1994). Wang \& Silk (1994) give an effective $Q$ parameter for this two-fluid instability such that $Q_{\text {eff }}=\alpha_{\text {eff }} Q$, where

$$
\alpha_{\mathrm{eff}}=\left(1+\frac{\mu_{*} \sigma_{g}}{\mu_{g} \sigma_{*}}\right)^{-1}
$$

In the limit of a low stellar surface density or large stellar velocity dispersion, we have $\alpha_{\text {eff }} \approx 1.0$, and $\alpha_{\text {eff }}$ gets smaller 

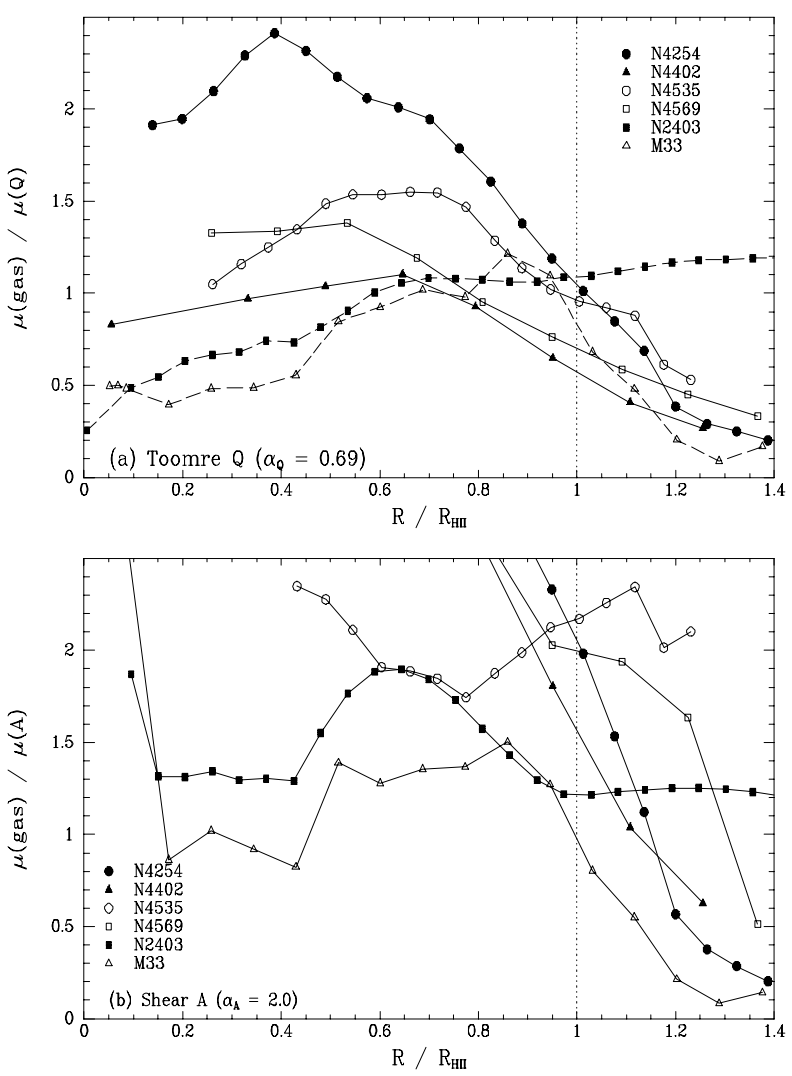

FIG. 13.-Comparison of the Toomre and shear instability parameters at the outer thresholds. The text describes why these six galaxies are shown.

as the stellar disk becomes more unstable relative to the gaseous disk. Following Wang \& Silk (1994), the parameters for the Milky Way disk yield $\alpha_{\text {eff }}=0.72$ in the solar neighborhood.

How much should we expect this parameter to vary over our sample? The gas disk is a smaller fraction of the total gas mass in the earlier type galaxies, so the stellar disks are expected to have a more important influence on the stability of those disks. Scaling to the disk of the Milky Way (e.g., Kenney \& Young 1989), the measured rotation velocities of these disks at $R_{\mathrm{H} \mathrm{II}}$ imply average surface densities of $15-300$ $M_{\odot} \mathrm{pc}^{-2}$. If the other parameters are held at their solar neighborhood values, then the highest surface density disks should become unstable at a value of $\mu_{\text {gas }} / \mu_{\text {crit }}$ that is $\sim 25 \%$ lower than $\alpha_{Q}$ in the lower density disks. In other words, we would not be surprised to see $\alpha_{Q}$ values ranging from 0.25 to 1.0 across our sample. Better measurements of the stellar velocity dispersion and surface density would be needed to test whether the lack of such a trend poses a problem for the gravitational instability model.

\subsection{Consequences for Star Formation Prescriptions}

We have presented a picture where a single parameter, the gas surface density relative to the critical density for gravitational instability, determines whether the disk forms stars. Evolution in the cosmic SFR is likely driven, at least in part, by the evolution in the rate of galaxy-galaxy interactions with look-back time (e.g., Tan, Silk, \& Balland 1999). In our star formation recipe, the environment influences the SFR indirectly through its impact on the largescale gas distribution. Including the star formation threshold in the recipe allows the SFR to react nonlinearly to small changes in the gas distribution.

Our $\mathrm{H} \alpha$ atlas demonstrates that the distribution of starforming regions in many disks is better described as asymmetric rather than axisymmetric. The correlations we find between local values of the instability parameter and the distribution of $\mathrm{H}$ II regions clearly indicate that the Toomre $Q$ parameter provides useful guidance about the location of star-forming regions in these disks. For example, in $\S 3.5 .1$, we found that disks are more unstable on the side with the higher SFR. Prior to that we showed that star formation at subcritical radii in NGC 2903, NGC 3031, and NGC 4548 is confined to the spiral arms. The average gas surface density was within a factor of 3 of the critical value in all three cases. The increase in gas surface density in the arms is expected to be larger than the increase in the epicyclic frequency $\kappa$ (and hence critical density) caused by unresolved, streaming motions (Rand 1993; Elmegreen 1994), so the arm gas may be supercritical locally. Since these features will not be resolved in cosmological simulations either, it would be reasonable to lower the $\alpha_{Q}$ value in equation (2) from 0.69 to $\sim 0.3$ in galaxies undergoing weak interactions to account for unresolved, supercritical structure in the gas distribution

The fraction of gas residing in subcritical regions of disks varies widely among the galaxies in our sample. Accounting for gas beyond the threshold radius produces the most significant correction to the global SFR. Among the spiral galaxies in our sample, the gas mass interior to the threshold radius $R_{\mathrm{H} \text { II }}$ varies from 0.14 to 0.94 of the total gas mass. Omitting the threshold radius from a model therefore leaves the global SFR uncertain by a factor of $\sim 7$. The median value of the gas fraction interior to $R_{\mathrm{H} \text { II }}$ is 0.6 . Ram pressure stripping has removed diffuse, atomic gas from at least these Virgo Cluster galaxies: NGC 4394, NGC 4402, NGC 4569, and NGC 4689 (Warmels 1988a, 1988b; Giovanelli \& Haynes 1983; Kenney \& Young 1989), yet a histogram of the gas mass fraction within $R_{\mathrm{H} \text { II }}$ for the Virgo subsample is indistinguishable from the histogram for the field subsample.

\section{SUMMARY}

Using a larger sample of spiral galaxies than previous studies, we tested the thesis that widespread star formation occurs where the gas disk is unstable to axisymmetric perturbations. We located the edge of the star-forming disk using our new $\mathrm{H} \alpha$ photometry, measured the gas surface density there, and derived the critical surface density for gravitational instability using the Toomre $Q$ criterion. Our results confirm previous work by K89, which found that the threshold gas density varied by at least an order of magnitude among spiral galaxies but that the ratio of the gas density to the critical density was much more uniform. Among 26 galaxies with well-defined thresholds, we found that the median ratio of gas surface density to critical density is $\alpha_{Q}=0.69 \pm 0.2$, with $\alpha\left(R_{\mathrm{H} \text { II }}\right)$ defined by equation (2) and an assumed gas velocity dispersion of $6 \mathrm{~km} \mathrm{~s}^{-1}$. At the thresholds, the gas is primarily atomic (molecular) in the disks with the lowest (highest) gas surface density. These results confirm the surprising accuracy with which a simple stability criterion describes the extent of the star-forming disk in large, objectively defined samples of galaxies.

This work also exposes the limitations of that simple model. The azimuthally averaged, threshold values of the 
instability parameter, $\alpha\left(R_{\mathrm{H}}\right)$, range from 0.3 to 1.2 . A large part of that dispersion is due to uncertainties associated with asymmetric gas distributions, asymmetric mass distributions, and the extrapolation of the molecular gas surface density profiles. We demonstrated that the scatter in $\alpha\left(R_{\mathrm{H} \mathrm{II}}\right)$ values is significantly reduced when $R_{\mathrm{H} \mathrm{II}}$ and $\alpha\left(R_{\mathrm{H} \mathrm{II}}\right)$ are fitted as functions of position angle. This result demonstrates that the simple Toomre $Q$ stability criterion is useful for describing the distribution of star-forming regions even in disks with quite nonaxisymmetric gas distributions. The weak trend toward lower values of $\alpha\left(R_{\mathrm{H} \text { II }}\right)$ in Sab-Sbc galaxies, however, is not easily explained by any of the above systematics. These early-type spiral galaxies tend to have more massive stellar disks, so the lower values of $\alpha_{Q}$ would be consistent with the predicted destabilizing effect of a stellar disk on the gas disk. We find several sharp thresholds in the inner disks that are well described by gravitational stability alone. Star formation is also suppressed in the centers of a few disks where the gas density is supercritical, but each of these disks has a strong stellar bar. More detailed analysis of the SFR in the inner region of disks requires higher spatial resolution (see Jogee 1999; Jogee, Kenney, \& Scoville 2001). We conclude that streaming motions in the disk and the gravitational attraction of the stellar disk require small corrections to the simple instability parameter. Much of the uncertainty in current estimates of the instability parameter, however, should be resolved with complete two-dimensional mapping of the gas surface density and velocity field (e.g., Thornley et al. 1999).

One clear failure of the gravitational threshold model remains two subcritical disks with widespread star formation in the inner disk: NGC 2403 and M33. Both are lowmass disks with low shear rates (i.e., slowly rising rotation curves). Elmegreen (1993) and HEB98 have suggested that star formation is limited by the time available for clouds to grow via inelastic collisions (in the destructive presence of disk shear) rather than cloud formation, which they argue happens easily via magnetic instabilities. We demonstrate that their shear criterion offers an explanation for the high SFRs in the inner disks of NGC 2403 and M33 but find that it does not consistently predict the outer (or inner) threshold radii. The vertical structure of these disks is also unusual in that the infall rate is high, and the high SFRs may be indirectly related to this transitional state. Since the SFR depends more naturally on gas volume density rather than surface density, the impact of the vertical structure of gas disks on the star formation law remains an important issue in a more general sense. Unfortunately, radial variation in the gas velocity dispersion remains controversial because of the difficulty of the measurement (e.g., Ferguson et al. 1998; Sellwood \& Balbus 1999). Among the galaxies in our sample, the intrinsic dispersion in the threshold value of the instability parameter is small for a constant velocity dispersion, leaving little motivation for strong variation in velocity dispersion with radius. Given the difficulties in measuring velocity dispersions accurately, the issue could be settled by comparing the threshold radii reported here to models of the transition radius from disk self-gravity to halo self-gravity.

The utility of the Toomre $Q$ criterion extends beyond axisymmetric perturbations. Previous studies of subcritical disks have focused on low surface brightness galaxies, which also have lower than average gas surface density. We showed that star formation is similarly suppressed in disks with normal gas surface density if the rotation speed is fast enough to stabilize the disk. As the gas density approaches the critical density for instability, more isolated $\mathrm{H}$ II regions appear in the subcritical disks. Similarly, in star-forming disks, $\mathrm{H}$ II regions are found outside the threshold radius most frequently when the gas density in the outer disk is near (i.e., within a factor of roughly 3 ) the critical density. Star formation is sometimes confined to a spiral density wave in these subcritical environments, and the corotation radius of the spiral pattern may describe the outer edge of the star formation activity. This result seems to support the view advanced by Rudnick, Rix, \& Kennicutt (2000) that tidal interactions impart a significant boost to the SFR in disk galaxies.

Star formation thresholds in the outer disk change one's view of disk star formation in two ways. First, in a typical disk today, roughly $40 \%$ of the gas mass is not included in the empirical relation that defines the global Schmidt law for the SFR, and the analogous correction should be made when global star formation laws are applied in simulations. Second, disks are clearly not perfectly self-regulated if their outer disks are not forming stars. Significant velocity dispersions in these regions imply that a physical process other than feedback from massive stars contributes to disk heating (Sellwood \& Balbus 1999). Because of the threshold effect in the star formation law, the central density and angular momentum of the halo are clearly of fundamental importance to the star formation history of a disk galaxy (Dalcanton, Spergel, \& Summers 1997; Mo, Mao, \& White 1998; Mao, Mo, \& White 1998).

We thank Rene Walterbos for making the KPNO 36 inch data available and Jean Turner and Pat Crosthwaite for providing a CO map of NGC 5236 in advance of publication. We thank Bruce Elmegreen, Sharda Jogee, and Nick Scoville for their comments on a draft of the paper and enlightening discussions. The comments of an anonymous referee were also appreciated. This research has made use of the NASA/IPAC Extragalactic Database (NED), which is operated by the Jet Propulsion Laboratory, California Institute of Technology, under contract with the National Aeronautics and Space Administration. C. L. M. acknowledges support from a Sherman Fairchild fellowship. R. C. K. gratefully acknowledges the support of NSF grant AST-9900789.

\section{REFERENCES}

Adler, D. S., \& Westpfahl, D. J. 1996, AJ, 111, 735

Bloemen, J. B. G. M., et al. 1986, A\&A, 154, 25

Bosma, A. 1981, AJ, 86, 1791

Bosma, A., Goss, W. M., \& Allen, R. J. 1981, A\&A, 93, 106

Bosma, A., van der Hulst, J. M., \& Sullivan, W. T., III 1977, A\&A, 57, 373

Braun, R., Walterbos, R. A. M., Kennicutt, R. C., \& Tacconi, L. J. 1994, ApJ, 420, 558

Carignan, C., Charbonneau, P., Boulanger, F., \& Viallefond, F. 1990,

A\&A, 234, 43
Cayatte, V., van Gorkom, J. H., Balkowski, C., \& Kotanyi, C. 1990, AJ, 100,604

Dalcanton, J. J., Spergel, D. N., \& Summers, F. J. 1997, ApJ, 482, 659

Dickey, J. M., Hanson, M. M., \& Helou, G. 1990, ApJ, 352, 522

Elmegreen, B. 1987, ApJ, 312, 626

1991, ApJ, 378, 139 , in Star Formation, Galaxies, and the Interstellar Medium,

ed. J. Franco, F. Ferrini, \& G. Tenorio-Tagle (Cambridge: Cambridge

Univ. Press), 337 
Elmegreen, B. 1994, ApJ, 433, 39 . 1995, MNRAS, 275, 944

Elmegreen, B., \& Parravano, A. 1994, ApJ, 435, L121

Federman, S. R., Glassgold, A. E., \& Kwan, J. 1979, ApJ, 227, 466

Ferguson, A., Wyse, R. F. G., Gallagher, J. S., \& Hunter, D. A. 1998, ApJ, 506, L19

Ferrarese, L., et al. 1996, ApJ, 464, 568

Freedman, W. L., et al. 1994a, ApJ, 427, 628 1994b, Nature, 371, 757

Gallagher, J. S., III, \& Hunter, D. A. 1984, ARA\&A, 22, 37

Gerin, M., Casoli, F., \& Combes, F. 1991, A\&A, 251, 32

Giovanelli, R., \& Haynes, M. P. 1983, AJ, 88, 881

Goldreich, P., \& Lynden-Bell, D. 1965, MNRAS, 130, 97

Graham, J. A., et al. 1999, ApJ, 516, 626

Gu, Q.-S., et al. 1996, A\&A, 314, 18

Guhathakurta, P., van Gorkom, J. H., Kotanyi, C. G., \& Balkowski, C. 1988, AJ, 96, 851

Hodge, P. W., \& Kennicutt, R. C. 1983, ApJ, 267, 563

Hughes, S. M. G., et al. 1998, ApJ, 501, 32

Hunter, D., Elmegreen, B. G., \& Baker, A. L. 1998, ApJ, 493, 595 (HEB98)

Hunter, D. A., \& Plummer, J. D. 1996, ApJ, 462, 732

Jog, C. J., \& Solomon, P. M. 1984a, ApJ, 276, 114 1984b, ApJ, 276, 127

Jogee, S. 1999, Ph.D. thesis, Yale Univ.

Jogee, S., Kenney, J. D. P., \& Scoville, N. 2001, ApJ, submitted

Kamphuis, J., \& Briggs, F. 1992, A\&A, 253, 335

Kelson, D. D., et al. 1996, ApJ, 463, 26

Kenney, J. D. P., Scoville, N. Z., \& Wilson, C. D. 1991, ApJ, 366, 432

Kenney, J. D., \& Young, J. S. 1988, ApJS, 66, 261 1989, ApJ, 344, 171

Kennicutt, R. C. 1989, ApJ, 344, 685 (K89) 1998, ApJ, 498, 541

Kennicutt, R. C., \& Kent, S. M. 1983, AJ, 88, 1094

Kennicutt, R. C., Tamblyn, P., \& Congdon, C. E. 1994, ApJ, 435, 22

Lelievre, M., \& Roy, J.-R. 2000, AJ, 120, 1306

Lord, S. D., \& Young, J. S. Y. 1990, ApJ, 356, 135

Macri, L. M., et al. 1999, ApJ, 521, 155

Mao, S., Mo, H. J., \& White, S. D. M. 1998, MNRAS, 297, L71

McCall, M. L., Rybski, P. M., \& Shields, G. A. 1985, ApJS, 57, 1

Miller, J. S., \& Mathews, W. G. 1972, ApJ, 172, 593

Mo, H. J., Mao, S., \& White, S. D. M. 1998, MNRAS, 295, 319

Newton, K. 1980, MNRAS, 190, 689

Pisano, D. J., Wilcots, E. M., \& Elmegreen, B. G. 1998, AJ, 115, 975 2000, AJ, 120, 763

Quirk, W. J. 1972, ApJ, 176, L9

Rand, R. J. 1993, ApJ, 410, 68

Rots, A. H. 1975, A\&A, 45, 43

Rubin, V. C., Waterman, A. H., \& Kenney, D. P. 1999, AJ, 118, 236

Rudnick, G., Rix, H.-W., \& Kennicutt, R. C. 2000, ApJ, 538, 569
Sage, L. J., \& Westpfahl, D. J. 1991, A\&A, 242, 371

Saha, A., et al. 1995, ApJ, 438, 8

Sancisi, R., Fraternali, F. Oosterloo, T. \& Moorsel, G. 2000, in Gas and Galaxy Evolution, ed. J. E. Hibbard, M. P. Rupen, \& J. H. van Gorkom (San Francisco: ASP), in press

Schaap, W. E., Sancisi, R., \& Swaters, R. A. 2000, A\&A, 356, L49

Schaller, G., Schaerer, D., Meynet, G., \& Maeder, A. 1992, A\&AS, 96, 269

Schmidt, M. 1959, ApJ, 129, 243

Sellwood, J. A., \& Balbus, S. A. 1999, ApJ, 511, 660

Shostak, G. S., \& van der Kruit, P. C. 1984, A\&A, 132, 20

Silbermann, N. A., et al. 1996, ApJ, 470, 1

Silk, J. 1997, ApJ, 481, 703

Skillman, E. D. 1987, in Star Formation in Galaxies, ed. C. J. Lonsdale Persson (NASA Conf. Pub. CP-2466), 263

Sofue, Y. 1996, ApJ, 458, 120

. 1997, PASJ, 49, 17

Spitzer, L. 1968, Diffuse Matter in Space (New York: Wiley)

Tacconi, L. J., \& Young, J. S. 1986, ApJ, 308, 600 1989, ApJS, 71, 455

Tan, J. C., Silk, J., \& Balland, C. 1999, ApJ, 522, 579

Thornley, M., et al. 1999, Ap\&SS, 269, 391

Thornley, M. D., \& Wilson, C. D. 1995, ApJ, 447, 616

Tilanus, R. P. J., \& Allen, R. J. 1991, A\&A, 244, 8 1993, A\&A, 274, 707

Toomre, A. 1964, ApJ, 139, 1217

Valluri, M., \& Jog, C. J. 1990, ApJ, 357, 367

van der Hulst, J. M., et al. 1993, AJ, 106, 548

van der Hulst, J. M., Kennicutt, R. C., Crane, P. C., \& Rots, A. H. 1988, A\&A, 195, 38

van der Kruit, P. C., \& Searle, L. 1981a, A\&A, 95, 105

- 1981b, A\&A, 95, 116 1982a, A\&A, 110, 61 1982b, A\&A, 110, 79

van der Kruit, P. C., \& Shostak, G. S. 1984, A\&A, 134, 258

van Zee, L., Haynes, M. P., Salzer, J. J., \& Broeils, A. H. 1996, AJ, 112, 129 1997, AJ, 113, 1618

Vollmer, B., Cayatte, V., Boselli, A., Balkowski, C., \& Duschl, W. J. 1999, A\&A, 349, 411

Wang, B., \& Silk, J. 1994, ApJ, 427, 759

Warmels, R. H. 1988a, A\&AS, 72, 19 .1988b, A\&AS, 72, 57 1988c, A\&AS, 72, 427

Webster, B. L., \& Smith, M. G. 1983, MNRAS, 204, 743

Westpfal, D. J. 1998, ApJS, 115, 203

Wevers, B. M. H. R., van der Kruit, P. C., \& Allen, R. J. 1986, A\&AS, 66, 505

Wilson, C. D. 1995, ApJ, 448, L97

Wong, T., \& Blitz, L. 2000, ApJ, 540, 771

Young, J. S., et al. 1995, ApJS, 98, 219

Zaritsky, D., Kennicutt, R. C., \& Huchra, J. 1994, ApJ, 420, 87 